Supporting Information for

\title{
Direct Tyrosine Oxidation using the MLCT Excited States of Rhenium Polypyridyl Complexes
}

\author{
Steven Y. Reece and Daniel G. Nocera * \\ Department of Chemistry, 6-335, Massachusetts Institute of Technology, 77 Massachusetts \\ Avenue, Cambridge, MA 02139-4307
}

Index

Page

X-ray crystal structure data for $\left[\mathrm{Re}(\mathrm{phen})(\mathrm{PP}-\mathrm{Bn})(\mathrm{CO})_{2}\right]\left(\mathrm{PF}_{6}\right) \cdot \mathrm{CDCl}_{3}$.

X-ray crystal structure data for $\left[\mathrm{Re}(\mathrm{phen})(\mathrm{dppe})(\mathrm{CO})_{2}\right]\left(\mathrm{PF}_{6}\right)$.

S14-S23

Spectroelectrochemical data for $\operatorname{Re}(\mathrm{bpy}-\mathrm{Y}-\mathrm{O} t \mathrm{Bu})(\mathrm{CO})_{3}(\mathrm{CN})$ 


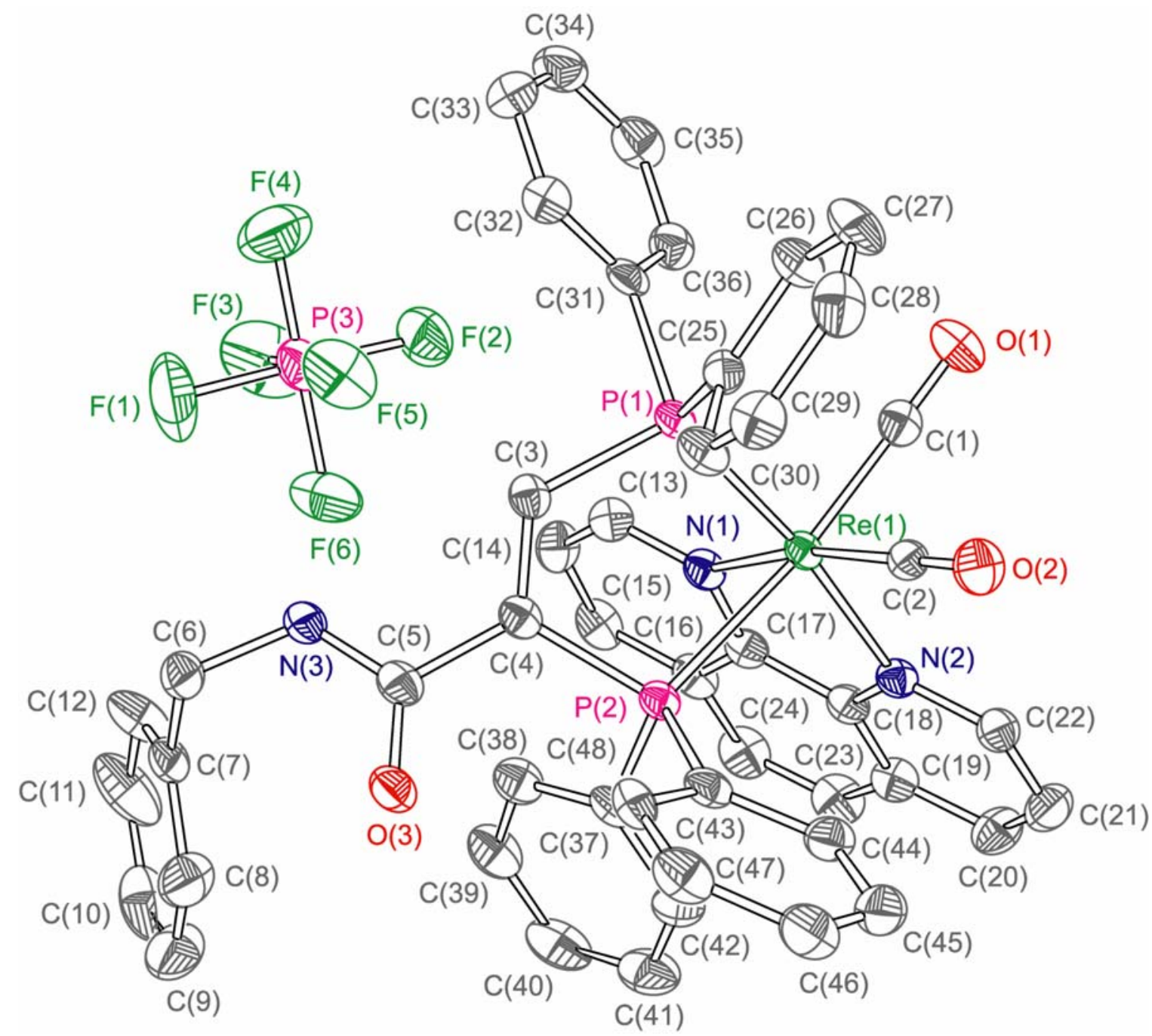

Figure S1. Atom labeling scheme for $\left[\mathrm{Re}(\mathrm{phen})(\mathrm{PP}-\mathrm{Bn})(\mathrm{CO})_{2}\right]\left(\mathrm{PF}_{6}\right) ; \mathrm{CDCl}_{3}$ not shown. 
Table S1. Crystal data and structure refinement for $\left[\mathrm{Re}(\mathrm{phen})(\mathrm{PP}-\mathrm{Bn})(\mathrm{CO})_{2}\right]\left(\mathrm{PF}_{6}\right) \cdot \mathrm{CDCl}_{3}$.

\begin{tabular}{ll}
\hline Empirical formula & $\mathrm{C}_{49} \mathrm{H}_{37} \mathrm{DCl}_{3} \mathrm{~F}_{6} \mathrm{~N}_{3} \mathrm{O}_{3} \mathrm{P}_{3} \mathrm{Re}$ \\
Formula weight & 1217.33 \\
$\mathrm{~T}(\mathrm{~K})$ & $150(2)$ \\
$\lambda(\AA)$ & 0.71073 \\
Crystal system & Triclinic \\
Space group & $P \overline{1}$ \\
$a(\AA)$ & $13.1590(14)$ \\
$b(\AA)$ & $13.9281(15)$ \\
$c(\AA)$ & $15.1161(16)$ \\
$\alpha\left(^{\circ}\right)$ & $73.916(2)$ \\
$\beta\left(^{\circ}\right)$ & $64.282(2)$ \\
$\gamma\left({ }^{\circ}\right)$ & $83.849(2)$ \\
$V\left(\AA^{3}\right)$ & $2398.1(4)$ \\
$Z$ & 2 \\
$\rho_{\text {calcd }}\left(\mathrm{Mg} / \mathrm{m}^{3}\right)$ & 1.683 \\
$\mathrm{Abs}$ coeff $\left(\mathrm{mm}{ }^{-1}\right)$ & 2.870 \\
$\theta$ range for data collection $\left(^{\circ}\right)$ & 1.52 to 23.27 \\
Index ranges & $-14 \leq h \leq 14,-15 \leq k \leq 11,-16 \leq \ell \leq 11$ \\
Reflections collected & 13421 \\
Independent reflns $\left(R_{\text {int }}\right)$ & $6833(0.0316)$ \\
Completeness to $\theta_{\text {max }}$ & $98.7 \%$ \\
Data/restraints/parameters & $6833 / 0 / 613$ \\
$\mathrm{GOF}^{a}$ on $F^{2}$ & 1.038 \\
$\mathrm{R} 1^{b}$ & 0.0348 \\
wR2 & 0.0904 \\
Largest diff. peak, hole $^{c}$ & $1.053,-1.688 \mathrm{e} \AA^{-3}$ \\
\hline
\end{tabular}

${ }^{\mathrm{a}} \mathrm{GOF}=\left(\Sigma w\left(F_{\mathrm{o}}^{2}-F_{\mathrm{c}}{ }^{2}\right)^{2} /(n-p)\right)^{1 / 2}$ where $n$ is the number of data and $p$ is the number of parameters refined. ${ }^{b} R 1=\Sigma|| F_{\mathrm{o}}-\left|F_{\mathrm{c}}\right||/ \Sigma| F_{\mathrm{o}} \mid \cdot{ }^{c}{ }^{c} \mathrm{w} R 2=\left(\Sigma\left(w\left(F_{\mathrm{o}}{ }^{2}-F_{\mathrm{c}}{ }^{2}\right)^{2}\right) / \Sigma\left(w\left(F_{\mathrm{o}}{ }^{2}\right)^{2}\right)\right)^{1 / 2}$. 
Table S2. Atomic coordinates $\left(\times 10^{4}\right)$ and equivalent isotropic displacement parameters $\left(\AA^{2}\right.$ $\times 10^{3}$ ) for $\left[\mathrm{Re}(\mathrm{phen})(\mathrm{PP}-\mathrm{Bn})(\mathrm{CO})_{2}\right]\left(\mathrm{PF}_{6}\right) \cdot \mathrm{CDCl}_{3}$.

\begin{tabular}{|c|c|c|c|c|}
\hline Atom & $\mathrm{x}$ & $\mathrm{y}$ & $\mathrm{z}$ & $\mathrm{U}_{\mathrm{eq}}$ \\
\hline $\operatorname{Re}(1)$ & $7184(1)$ & $5961(1)$ & $5697(1)$ & $19(1)$ \\
\hline $\mathrm{P}(1)$ & $6853(1)$ & $5498(1)$ & $7422(1)$ & $20(1)$ \\
\hline $\mathrm{P}(2)$ & $5844(1)$ & $7283(1)$ & $6285(1)$ & $20(1)$ \\
\hline $\mathrm{P}(3)$ & $9030(1)$ & $7914(1)$ & $8170(1)$ & $35(1)$ \\
\hline $\mathrm{N}(1)$ & $8475(3)$ & $7161(2)$ & $5100(2)$ & $24(1)$ \\
\hline $\mathrm{N}(2)$ & $7462(3)$ & $6583(2)$ & $4120(2)$ & $22(1)$ \\
\hline $\mathrm{N}(3)$ & $5905(3)$ & $8507(2)$ & $8390(2)$ & $26(1)$ \\
\hline $\mathrm{O}(1)$ & $8818(3)$ & $4202(2)$ & $5261(2)$ & $40(1)$ \\
\hline $\mathrm{O}(2)$ & $5325(2)$ & $4498(2)$ & $6177(2)$ & $34(1)$ \\
\hline $\mathrm{O}(3)$ & $4541(2)$ & $8675(2)$ & $7825(2)$ & $29(1)$ \\
\hline $\mathrm{C}(1)$ & $8230(3)$ & $4884(3)$ & $5396(3)$ & $26(1)$ \\
\hline$C(2)$ & $6022(3)$ & $5056(3)$ & $6012(3)$ & $23(1)$ \\
\hline$C(3)$ & $6239(3)$ & $6551(3)$ & 7983(3) & $22(1)$ \\
\hline$C(4)$ & $5841(3)$ & $7341(3)$ & 7491(3) & $22(1)$ \\
\hline$C(5)$ & $5368(3)$ & $8236(3)$ & $7918(3)$ & $25(1)$ \\
\hline$C(6)$ & $5537(4)$ & $9365(3)$ & $8844(3)$ & $31(1)$ \\
\hline$C(7)$ & $5943(4)$ & $10358(3)$ & $8065(3)$ & $29(1)$ \\
\hline $\mathrm{C}(8)$ & $5270(5)$ & $10895(3)$ & $7619(4)$ & $48(1)$ \\
\hline$C(9)$ & $5654(6)$ & $11810(4)$ & $6890(4)$ & $62(2)$ \\
\hline$C(10)$ & $6702(6)$ & $12182(4)$ & $6630(4)$ & $61(2)$ \\
\hline$C(11)$ & $7368(5)$ & 11657(4) & $7094(5)$ & $61(2)$ \\
\hline$C(12)$ & 6991(4) & 10739(3) & $7809(4)$ & $46(1)$ \\
\hline$C(13)$ & $8955(3)$ & 7463(3) & $5588(3)$ & $28(1)$ \\
\hline$C(14)$ & $9674(4)$ & $8296(3)$ & $5158(3)$ & $33(1)$ \\
\hline$C(15)$ & $9918(3)$ & $8837(3)$ & $4182(3)$ & $33(1)$ \\
\hline$C(16)$ & $9450(3)$ & $8533(3)$ & $3623(3)$ & $29(1)$ \\
\hline$C(17)$ & $8727(3)$ & $7690(3)$ & $4115(3)$ & $26(1)$ \\
\hline $\mathrm{C}(18)$ & $8197(3)$ & $7368(3)$ & $3591(3)$ & $24(1)$ \\
\hline$C(19)$ & $8463(3)$ & $7856(3)$ & $2561(3)$ & $30(1)$ \\
\hline$C(20)$ & $7938(4)$ & $7488(3)$ & $2076(3)$ & $36(1)$ \\
\hline $\mathrm{C}(21)$ & $7202(4)$ & $6702(3)$ & $2621(3)$ & $36(1)$ \\
\hline $\mathrm{C}(22)$ & $6973(3)$ & $6261(3)$ & $3638(3)$ & $26(1)$ \\
\hline$C(23)$ & $9232(4)$ & $8692(3)$ & 2074(3) & $38(1)$ \\
\hline$C(24)$ & $9686(4)$ & $9023(3)$ & $2585(3)$ & $37(1)$ \\
\hline$C(25)$ & $5783(3)$ & $4519(3)$ & $8237(3)$ & $22(1)$ \\
\hline
\end{tabular}




\begin{tabular}{|c|c|c|c|c|}
\hline$C(26)$ & $6101(4)$ & $3520(3)$ & $8394(3)$ & $30(1)$ \\
\hline$C(27)$ & $5303(4)$ & $2758(3)$ & $8896(3)$ & $35(1)$ \\
\hline $\mathrm{C}(28)$ & $4167(4)$ & 2977(3) & $9262(3)$ & $32(1)$ \\
\hline C(29) & $3834(4)$ & $3967(3)$ & $9123(3)$ & $31(1)$ \\
\hline$C(30)$ & $4639(3)$ & $4733(3)$ & $8605(3)$ & $28(1)$ \\
\hline$C(31)$ & $7978(3)$ & $5102(3)$ & $7845(3)$ & $24(1)$ \\
\hline$C(32)$ & $7735(4)$ & $4854(3)$ & $8877(3)$ & $32(1)$ \\
\hline$C(33)$ & $8589(4)$ & $4557(3)$ & $9196(4)$ & $41(1)$ \\
\hline$C(34)$ & $9676(4)$ & $4476(3)$ & $8501(4)$ & $41(1)$ \\
\hline$C(35)$ & $9931(4)$ & $4708(3)$ & $7479(4)$ & $37(1)$ \\
\hline$C(36)$ & $9085(3)$ & $5032(3)$ & $7141(3)$ & $30(1)$ \\
\hline$C(37)$ & $6207(3)$ & $8555(3)$ & $5472(3)$ & $23(1)$ \\
\hline$C(38)$ & $6873(4)$ & $9175(3)$ & $5603(3)$ & $32(1)$ \\
\hline$C(39)$ & $7244(4)$ & $10098(3)$ & $4914(4)$ & $39(1)$ \\
\hline$C(40)$ & $6978(4)$ & $10416(3)$ & $4092(4)$ & $45(1)$ \\
\hline$C(41)$ & $6326(4)$ & $9806(3)$ & $3962(3)$ & $43(1)$ \\
\hline$C(42)$ & $5942(4)$ & $8881(3)$ & $4640(3)$ & $32(1)$ \\
\hline$C(43)$ & $4388(3)$ & $7028(3)$ & $6561(3)$ & $24(1)$ \\
\hline$C(44)$ & $4202(4)$ & $6734(3)$ & $5829(3)$ & $28(1)$ \\
\hline$C(45)$ & $3128(4)$ & $6508(3)$ & $5992(3)$ & $34(1)$ \\
\hline$C(46)$ & $2209(4)$ & $6548(3)$ & $6901(4)$ & $36(1)$ \\
\hline$C(47)$ & $2389(3)$ & $6816(3)$ & $7637(3)$ & $33(1)$ \\
\hline $\mathrm{C}(48)$ & $3467(3)$ & $7060(3)$ & $7472(3)$ & $26(1)$ \\
\hline$C(49)$ & $7810(4)$ & $1110(3)$ & 551(4) & $40(1)$ \\
\hline $\mathrm{Cl}(1)$ & $8008(1)$ & $27(1)$ & $106(1)$ & $43(1)$ \\
\hline $\mathrm{Cl}(2)$ & $8975(1)$ & $1335(1)$ & $738(1)$ & $61(1)$ \\
\hline $\mathrm{Cl}(3)$ & $7547(2)$ & 2133(1) & $-288(1)$ & $72(1)$ \\
\hline $\mathrm{F}(1)$ & $8395(4)$ & $8575(3)$ & $8953(3)$ & $92(1)$ \\
\hline $\mathrm{F}(2)$ & $9623(2)$ & $7261(2)$ & $7382(2)$ & $54(1)$ \\
\hline $\mathrm{F}(3)$ & $10151(3)$ & $8548(3)$ & $7702(4)$ & $91(1)$ \\
\hline $\mathrm{F}(4)$ & $9428(3)$ & $7157(3)$ & $8945(3)$ & $71(1)$ \\
\hline $\mathrm{F}(5)$ & $7894(2)$ & $7287(2)$ & $8609(2)$ & $56(1)$ \\
\hline $\mathrm{F}(6)$ & $8629(3)$ & $8684(2)$ & $7375(2)$ & $60(1)$ \\
\hline
\end{tabular}

${ }^{a} \mathrm{U}_{\mathrm{eq}}$ is defined as one third of the trace of the orthogonalized $\mathrm{U}_{\mathrm{ij}}$ tensor. 
Table S3. Bond lengths $(\AA)$ for $\left[\mathrm{Re}(\mathrm{phen})(\mathrm{PP}-\mathrm{Bn})(\mathrm{CO})_{2}\right]\left(\mathrm{PF}_{6}\right) \cdot \mathrm{CDCl}_{3}$.

\begin{tabular}{|c|c|c|c|}
\hline Bond & Length & Bond & Length \\
\hline $\operatorname{Re}(1)-C(2)$ & $1.899(4)$ & $\mathrm{C}(15)-\mathrm{C}(16)$ & $1.406(6)$ \\
\hline $\operatorname{Re}(1)-C(1)$ & $1.936(4)$ & $C(16)-C(17)$ & $1.407(6)$ \\
\hline $\mathrm{Re}(1)-\mathrm{N}(2)$ & $2.175(3)$ & $C(16)-C(24)$ & $1.433(6)$ \\
\hline $\operatorname{Re}(1)-\mathrm{N}(1)$ & $2.221(3)$ & $\mathrm{C}(17)-\mathrm{C}(18)$ & $1.437(6)$ \\
\hline $\operatorname{Re}(1)-\mathrm{P}(1)$ & $2.3552(10)$ & $\mathrm{C}(18)-\mathrm{C}(19)$ & $1.414(6)$ \\
\hline $\operatorname{Re}(1)-\mathrm{P}(2)$ & $2.4787(10)$ & $C(19)-C(20)$ & $1.415(6)$ \\
\hline $\mathrm{P}(1)-\mathrm{C}(25)$ & $1.827(4)$ & $\mathrm{C}(19)-\mathrm{C}(23)$ & $1.433(6)$ \\
\hline $\mathrm{P}(1)-\mathrm{C}(31)$ & $1.832(4)$ & $\mathrm{C}(20)-\mathrm{C}(21)$ & $1.360(6)$ \\
\hline $\mathrm{P}(1)-\mathrm{C}(3)$ & $1.835(4)$ & $\mathrm{C}(21)-\mathrm{C}(22)$ & $1.395(6)$ \\
\hline$P(2)-C(43)$ & $1.827(4)$ & $\mathrm{C}(23)-\mathrm{C}(24)$ & $1.350(7)$ \\
\hline$P(2)-C(37)$ & $1.828(4)$ & $\mathrm{C}(25)-\mathrm{C}(30)$ & $1.390(6)$ \\
\hline $\mathrm{P}(2)-\mathrm{C}(4)$ & $1.845(4)$ & $C(25)-C(26)$ & $1.395(5)$ \\
\hline $\mathrm{P}(3)-\mathrm{F}(3)$ & $1.576(3)$ & $C(26)-C(27)$ & $1.380(6)$ \\
\hline $\mathrm{P}(3)-\mathrm{F}(4)$ & $1.577(3)$ & $\mathrm{C}(27)-\mathrm{C}(28)$ & $1.383(6)$ \\
\hline $\mathrm{P}(3)-\mathrm{F}(2)$ & $1.581(3)$ & $\mathrm{C}(28)-\mathrm{C}(29)$ & $1.389(6)$ \\
\hline $\mathrm{P}(3)-\mathrm{F}(1)$ & $1.590(3)$ & $\mathrm{C}(29)-\mathrm{C}(30)$ & $1.390(6)$ \\
\hline $\mathrm{P}(3)-\mathrm{F}(5)$ & $1.592(3)$ & $\mathrm{C}(31)-\mathrm{C}(32)$ & $1.395(6)$ \\
\hline $\mathrm{P}(3)-\mathrm{F}(6)$ & $1.609(3)$ & $\mathrm{C}(31)-\mathrm{C}(36)$ & $1.396(6)$ \\
\hline $\mathrm{N}(1)-\mathrm{C}(13)$ & $1.325(5)$ & $\mathrm{C}(32)-\mathrm{C}(33)$ & $1.388(6)$ \\
\hline $\mathrm{N}(1)-\mathrm{C}(17)$ & $1.372(5)$ & $\mathrm{C}(33)-\mathrm{C}(34)$ & $1.374(7)$ \\
\hline $\mathrm{N}(2)-\mathrm{C}(22)$ & $1.341(5)$ & $\mathrm{C}(34)-\mathrm{C}(35)$ & $1.378(7)$ \\
\hline $\mathrm{N}(2)-\mathrm{C}(18)$ & $1.356(5)$ & $C(35)-C(36)$ & $1.401(6)$ \\
\hline $\mathrm{N}(3)-\mathrm{C}(5)$ & $1.340(5)$ & $\mathrm{C}(37)-\mathrm{C}(42)$ & $1.393(6)$ \\
\hline $\mathrm{N}(3)-\mathrm{C}(6)$ & $1.474(5)$ & $\mathrm{C}(37)-\mathrm{C}(38)$ & $1.400(6)$ \\
\hline $\mathrm{O}(1)-\mathrm{C}(1)$ & $1.164(5)$ & $\mathrm{C}(38)-\mathrm{C}(39)$ & $1.389(6)$ \\
\hline $\mathrm{O}(2)-\mathrm{C}(2)$ & $1.163(5)$ & $\mathrm{C}(39)-\mathrm{C}(40)$ & $1.380(7)$ \\
\hline $\mathrm{O}(3)-\mathrm{C}(5)$ & $1.234(5)$ & $\mathrm{C}(40)-\mathrm{C}(41)$ & $1.375(7)$ \\
\hline$C(3)-C(4)$ & $1.342(5)$ & $\mathrm{C}(41)-\mathrm{C}(42)$ & $1.387(6)$ \\
\hline$C(4)-C(5)$ & $1.512(5)$ & $\mathrm{C}(43)-\mathrm{C}(48)$ & $1.393(6)$ \\
\hline$C(6)-C(7)$ & $1.515(6)$ & $C(43)-C(44)$ & $1.401(6)$ \\
\hline$C(7)-C(8)$ & $1.376(6)$ & $C(44)-C(45)$ & $1.380(6)$ \\
\hline$C(7)-C(12)$ & $1.383(7)$ & $C(45)-C(46)$ & $1.393(6)$ \\
\hline $\mathrm{C}(8)-\mathrm{C}(9)$ & $1.403(7)$ & $C(46)-C(47)$ & $1.382(6)$ \\
\hline C(9)-C(10) & $1.377(9)$ & $\mathrm{C}(47)-\mathrm{C}(48)$ & $1.393(6)$ \\
\hline $\mathrm{C}(10)-\mathrm{C}(11)$ & $1.381(9)$ & $\mathrm{C}(49)-\mathrm{Cl}(3)$ & $1.742(5)$ \\
\hline $\mathrm{C}(11)-\mathrm{C}(12)$ & $1.397(7)$ & $\mathrm{C}(49)-\mathrm{Cl}(2)$ & $1.749(5)$ \\
\hline
\end{tabular}




\begin{tabular}{llll}
$\mathrm{C}(13)-\mathrm{C}(14)$ & $1.395(6)$ & $\mathrm{C}(49)-\mathrm{Cl}(1)$ & $1.767(4)$ \\
$\mathrm{C}(14)-\mathrm{C}(15)$ & $1.368(6)$ & & \\
\hline
\end{tabular}


Table S4. Bond angles $\left({ }^{\circ}\right)$ for $\left[\mathrm{Re}(\mathrm{phen})(\mathrm{PP}-\mathrm{Bn})(\mathrm{CO})_{2}\right]\left(\mathrm{PF}_{6}\right) \cdot \mathrm{CDCl}_{3}$.

\begin{tabular}{|c|c|c|c|}
\hline Bond angle & Angle & Bond angle & Angle \\
\hline $\mathrm{C}(2)-\operatorname{Re}(1)-\mathrm{C}(1)$ & $87.22(16)$ & $C(12)-C(7)-C(6)$ & $119.9(4)$ \\
\hline $\mathrm{C}(2)-\operatorname{Re}(1)-\mathrm{N}(2)$ & $95.22(14)$ & $\mathrm{C}(7)-\mathrm{C}(8)-\mathrm{C}(9)$ & $120.4(5)$ \\
\hline $\mathrm{C}(1)-\operatorname{Re}(1)-\mathrm{N}(2)$ & $93.53(14)$ & $\mathrm{C}(10)-\mathrm{C}(9)-\mathrm{C}(8)$ & $119.9(5)$ \\
\hline $\mathrm{C}(2)-\operatorname{Re}(1)-\mathrm{N}(1)$ & $169.99(14)$ & $C(9)-C(10)-C(11)$ & $119.8(5)$ \\
\hline $\mathrm{C}(1)-\operatorname{Re}(1)-\mathrm{N}(1)$ & $96.63(14)$ & $\mathrm{C}(10)-\mathrm{C}(11)-\mathrm{C}(12)$ & $120.2(5)$ \\
\hline $\mathrm{N}(2)-\operatorname{Re}(1)-\mathrm{N}(1)$ & $75.37(12)$ & $\mathrm{C}(7)-\mathrm{C}(12)-\mathrm{C}(11)$ & $120.2(5)$ \\
\hline $\mathrm{C}(2)-\operatorname{Re}(1)-\mathrm{P}(1)$ & $89.07(11)$ & $\mathrm{N}(1)-\mathrm{C}(13)-\mathrm{C}(14)$ & $123.2(4)$ \\
\hline $\mathrm{C}(1)-\mathrm{Re}(1)-\mathrm{P}(1)$ & $92.50(12)$ & $\mathrm{C}(15)-\mathrm{C}(14)-\mathrm{C}(13)$ & $119.8(4)$ \\
\hline $\mathrm{N}(2)-\operatorname{Re}(1)-\mathrm{P}(1)$ & $172.77(8)$ & $C(14)-C(15)-C(16)$ & $119.2(4)$ \\
\hline $\mathrm{N}(1)-\operatorname{Re}(1)-\mathrm{P}(1)$ & 99.96(9) & $C(15)-C(16)-C(17)$ & $117.5(4)$ \\
\hline $\mathrm{C}(2)-\mathrm{Re}(1)-\mathrm{P}(2)$ & $93.48(12)$ & $\mathrm{C}(15)-\mathrm{C}(16)-\mathrm{C}(24)$ & $123.6(4)$ \\
\hline $\mathrm{C}(1)-\mathrm{Re}(1)-\mathrm{P}(2)$ & $173.16(12)$ & $\mathrm{C}(17)-\mathrm{C}(16)-\mathrm{C}(24)$ & $118.9(4)$ \\
\hline $\mathrm{N}(2)-\operatorname{Re}(1)-\mathrm{P}(2)$ & $93.19(8)$ & $\mathrm{N}(1)-\mathrm{C}(17)-\mathrm{C}(16)$ & $122.9(4)$ \\
\hline $\mathrm{N}(1)-\operatorname{Re}(1)-\mathrm{P}(2)$ & $83.80(8)$ & $\mathrm{N}(1)-\mathrm{C}(17)-\mathrm{C}(18)$ & $117.4(3)$ \\
\hline $\mathrm{P}(1)-\operatorname{Re}(1)-\mathrm{P}(2)$ & $80.71(3)$ & $\mathrm{C}(16)-\mathrm{C}(17)-\mathrm{C}(18)$ & $119.7(4)$ \\
\hline $\mathrm{C}(25)-\mathrm{P}(1)-\mathrm{C}(31)$ & $102.17(17)$ & $\mathrm{N}(2)-\mathrm{C}(18)-\mathrm{C}(19)$ & $122.9(4)$ \\
\hline $\mathrm{C}(25)-\mathrm{P}(1)-\mathrm{C}(3)$ & $101.86(17)$ & $\mathrm{N}(2)-\mathrm{C}(18)-\mathrm{C}(17)$ & $117.2(3)$ \\
\hline $\mathrm{C}(31)-\mathrm{P}(1)-\mathrm{C}(3)$ & $102.54(17)$ & $\mathrm{C}(19)-\mathrm{C}(18)-\mathrm{C}(17)$ & $119.9(4)$ \\
\hline $\mathrm{C}(25)-\mathrm{P}(1)-\operatorname{Re}(1)$ & $115.30(12)$ & $\mathrm{C}(18)-\mathrm{C}(19)-\mathrm{C}(20)$ & $117.4(4)$ \\
\hline $\mathrm{C}(31)-\mathrm{P}(1)-\operatorname{Re}(1)$ & $122.92(13)$ & $\mathrm{C}(18)-\mathrm{C}(19)-\mathrm{C}(23)$ & $118.7(4)$ \\
\hline $\mathrm{C}(3)-\mathrm{P}(1)-\operatorname{Re}(1)$ & $109.46(13)$ & $\mathrm{C}(20)-\mathrm{C}(19)-\mathrm{C}(23)$ & $123.9(4)$ \\
\hline $\mathrm{C}(43)-\mathrm{P}(2)-\mathrm{C}(37)$ & $107.58(17)$ & $\mathrm{C}(21)-\mathrm{C}(20)-\mathrm{C}(19)$ & $118.8(4)$ \\
\hline $\mathrm{C}(43)-\mathrm{P}(2)-\mathrm{C}(4)$ & $106.61(18)$ & $\mathrm{C}(20)-\mathrm{C}(21)-\mathrm{C}(22)$ & $120.7(4)$ \\
\hline $\mathrm{C}(37)-\mathrm{P}(2)-\mathrm{C}(4)$ & $104.56(17)$ & $\mathrm{N}(2)-\mathrm{C}(22)-\mathrm{C}(21)$ & $122.2(4)$ \\
\hline $\mathrm{C}(43)-\mathrm{P}(2)-\operatorname{Re}(1)$ & $114.11(12)$ & $\mathrm{C}(24)-\mathrm{C}(23)-\mathrm{C}(19)$ & $121.2(4)$ \\
\hline $\mathrm{C}(37)-\mathrm{P}(2)-\operatorname{Re}(1)$ & $116.44(12)$ & $\mathrm{C}(23)-\mathrm{C}(24)-\mathrm{C}(16)$ & $121.5(4)$ \\
\hline $\mathrm{C}(4)-\mathrm{P}(2)-\operatorname{Re}(1)$ & $106.68(12)$ & $\mathrm{C}(30)-\mathrm{C}(25)-\mathrm{C}(26)$ & $118.4(4)$ \\
\hline $\mathrm{F}(3)-\mathrm{P}(3)-\mathrm{F}(4)$ & $90.5(2)$ & $\mathrm{C}(30)-\mathrm{C}(25)-\mathrm{P}(1)$ & $121.3(3)$ \\
\hline $\mathrm{F}(3)-\mathrm{P}(3)-\mathrm{F}(2)$ & $89.9(2)$ & $\mathrm{C}(26)-\mathrm{C}(25)-\mathrm{P}(1)$ & $119.7(3)$ \\
\hline $\mathrm{F}(4)-\mathrm{P}(3)-\mathrm{F}(2)$ & $89.74(18)$ & $\mathrm{C}(27)-\mathrm{C}(26)-\mathrm{C}(25)$ & $121.1(4)$ \\
\hline $\mathrm{F}(3)-\mathrm{P}(3)-\mathrm{F}(1)$ & $91.5(2)$ & $\mathrm{C}(26)-\mathrm{C}(27)-\mathrm{C}(28)$ & $120.1(4)$ \\
\hline $\mathrm{F}(4)-\mathrm{P}(3)-\mathrm{F}(1)$ & $91.8(2)$ & $\mathrm{C}(27)-\mathrm{C}(28)-\mathrm{C}(29)$ & $119.6(4)$ \\
\hline $\mathrm{F}(2)-\mathrm{P}(3)-\mathrm{F}(1)$ & $177.9(2)$ & $\mathrm{C}(28)-\mathrm{C}(29)-\mathrm{C}(30)$ & $120.1(4)$ \\
\hline $\mathrm{F}(3)-\mathrm{P}(3)-\mathrm{F}(5)$ & $178.2(2)$ & $\mathrm{C}(29)-\mathrm{C}(30)-\mathrm{C}(25)$ & $120.6(4)$ \\
\hline $\mathrm{F}(4)-\mathrm{P}(3)-\mathrm{F}(5)$ & $91.16(19)$ & $\mathrm{C}(32)-\mathrm{C}(31)-\mathrm{C}(36)$ & $119.2(4)$ \\
\hline $\mathrm{F}(2)-\mathrm{P}(3)-\mathrm{F}(5)$ & $89.36(17)$ & $\mathrm{C}(32)-\mathrm{C}(31)-\mathrm{P}(1)$ & $120.4(3)$ \\
\hline
\end{tabular}




\begin{tabular}{lcll}
$\mathrm{F}(1)-\mathrm{P}(3)-\mathrm{F}(5)$ & $89.2(2)$ & $\mathrm{C}(36)-\mathrm{C}(31)-\mathrm{P}(1)$ & $120.4(3)$ \\
$\mathrm{F}(3)-\mathrm{P}(3)-\mathrm{F}(6)$ & $89.4(2)$ & $\mathrm{C}(33)-\mathrm{C}(32)-\mathrm{C}(31)$ & $120.3(4)$ \\
$\mathrm{F}(4)-\mathrm{P}(3)-\mathrm{F}(6)$ & $179.7(2)$ & $\mathrm{C}(34)-\mathrm{C}(33)-\mathrm{C}(32)$ & $120.3(4)$ \\
$\mathrm{F}(2)-\mathrm{P}(3)-\mathrm{F}(6)$ & $89.95(17)$ & $\mathrm{C}(33)-\mathrm{C}(34)-\mathrm{C}(35)$ & $120.3(4)$ \\
$\mathrm{F}(1)-\mathrm{P}(3)-\mathrm{F}(6)$ & $88.5(2)$ & $\mathrm{C}(34)-\mathrm{C}(35)-\mathrm{C}(36)$ & $120.2(4)$ \\
$\mathrm{F}(5)-\mathrm{P}(3)-\mathrm{F}(6)$ & $88.90(18)$ & $\mathrm{C}(31)-\mathrm{C}(36)-\mathrm{C}(35)$ & $119.7(4)$ \\
$\mathrm{C}(13)-\mathrm{N}(1)-\mathrm{C}(17)$ & $117.5(3)$ & $\mathrm{C}(42)-\mathrm{C}(37)-\mathrm{C}(38)$ & $118.7(4)$ \\
$\mathrm{C}(13)-\mathrm{N}(1)-\mathrm{Re}(1)$ & $128.5(3)$ & $\mathrm{C}(42)-\mathrm{C}(37)-\mathrm{P}(2)$ & $120.5(3)$ \\
$\mathrm{C}(17)-\mathrm{N}(1)-\mathrm{Re}(1)$ & $113.8(2)$ & $\mathrm{C}(38)-\mathrm{C}(37)-\mathrm{P}(2)$ & $120.3(3)$ \\
$\mathrm{C}(22)-\mathrm{N}(2)-\mathrm{C}(18)$ & $118.0(3)$ & $\mathrm{C}(39)-\mathrm{C}(38)-\mathrm{C}(37)$ & $119.7(4)$ \\
$\mathrm{C}(22)-\mathrm{N}(2)-\mathrm{Re}(1)$ & $125.9(3)$ & $\mathrm{C}(40)-\mathrm{C}(39)-\mathrm{C}(38)$ & $121.2(4)$ \\
$\mathrm{C}(18)-\mathrm{N}(2)-\mathrm{Re}(1)$ & $116.1(2)$ & $\mathrm{C}(41)-\mathrm{C}(40)-\mathrm{C}(39)$ & $119.1(4)$ \\
$\mathrm{C}(5)-\mathrm{N}(3)-\mathrm{C}(6)$ & $121.7(3)$ & $\mathrm{C}(40)-\mathrm{C}(41)-\mathrm{C}(42)$ & $121.0(4)$ \\
$\mathrm{O}(1)-\mathrm{C}(1)-\mathrm{Re}(1)$ & $176.4(4)$ & $\mathrm{C}(41)-\mathrm{C}(42)-\mathrm{C}(37)$ & $120.3(4)$ \\
$\mathrm{O}(2)-\mathrm{C}(2)-\mathrm{Re}(1)$ & $178.0(3)$ & $\mathrm{C}(48)-\mathrm{C}(43)-\mathrm{C}(44)$ & $118.4(4)$ \\
$\mathrm{C}(4)-\mathrm{C}(3)-\mathrm{P}(1)$ & $120.6(3)$ & $\mathrm{C}(48)-\mathrm{C}(43)-\mathrm{P}(2)$ & $124.0(3)$ \\
$\mathrm{C}(3)-\mathrm{C}(4)-\mathrm{C}(5)$ & $121.7(3)$ & $\mathrm{C}(44)-\mathrm{C}(43)-\mathrm{P}(2)$ & $117.5(3)$ \\
$\mathrm{C}(3)-\mathrm{C}(4)-\mathrm{P}(2)$ & $117.6(3)$ & $\mathrm{C}(45)-\mathrm{C}(44)-\mathrm{C}(43)$ & $120.9(4)$ \\
$\mathrm{C}(5)-\mathrm{C}(4)-\mathrm{P}(2)$ & $120.7(3)$ & $\mathrm{C}(44)-\mathrm{C}(45)-\mathrm{C}(46)$ & $120.5(4)$ \\
$\mathrm{O}(3)-\mathrm{C}(5)-\mathrm{N}(3)$ & $123.4(3)$ & $\mathrm{C}(47)-\mathrm{C}(46)-\mathrm{C}(45)$ & $119.0(4)$ \\
$\mathrm{O}(3)-\mathrm{C}(5)-\mathrm{C}(4)$ & $120.8(3)$ & $\mathrm{C}(46)-\mathrm{C}(47)-\mathrm{C}(48)$ & $120.9(4)$ \\
$\mathrm{N}(3)-\mathrm{C}(5)-\mathrm{C}(4)$ & $115.8(3)$ & $\mathrm{C}(43)-\mathrm{C}(48)-\mathrm{C}(47)$ & $120.3(4)$ \\
$\mathrm{N}(3)-\mathrm{C}(6)-\mathrm{C}(7)$ & $112.7(3)$ & $\mathrm{Cl}(3)-\mathrm{C}(49)-\mathrm{Cl}(2)$ & $111.3(3)$ \\
$\mathrm{C}(8)-\mathrm{C}(7)-\mathrm{C}(12)$ & $119.5(4)$ & $\mathrm{Cl}(3)-\mathrm{C}(49)-\mathrm{Cl}(1)$ & $110.3(3)$ \\
$\mathrm{C}(8)-\mathrm{C}(7)-\mathrm{C}(6)$ & $120.6(4)$ & $\mathrm{Cl}(2)-\mathrm{C}(49)-\mathrm{Cl}(1)$ & $110.8(3)$ \\
\hline & & & \\
& & &
\end{tabular}


Table S5. Anisotropic displacement parameters $\left(\AA^{2} \times 10^{3}\right)$ for $[\mathrm{Re}(\mathrm{phen})(\mathrm{PP}-$ $\left.\mathrm{Bn})(\mathrm{CO})_{2}\right]\left(\mathrm{PF}_{6}\right) \cdot \mathrm{CDCl}_{3}$.

\begin{tabular}{|c|c|c|c|c|c|c|}
\hline Atom & $\mathrm{U}_{11}$ & $\mathrm{U}_{22}$ & $\mathrm{U}_{33}$ & $\mathrm{U}_{23}$ & $\mathrm{U}_{13}$ & $\mathrm{U}_{12}$ \\
\hline $\operatorname{Re}(1)$ & $19(1)$ & $14(1)$ & $21(1)$ & $-3(1)$ & $-7(1)$ & $-2(1)$ \\
\hline $\mathrm{P}(1)$ & $22(1)$ & $14(1)$ & $22(1)$ & $-3(1)$ & $-9(1)$ & $0(1)$ \\
\hline $\mathrm{P}(2)$ & $20(1)$ & $15(1)$ & $23(1)$ & $-3(1)$ & $-8(1)$ & $-1(1)$ \\
\hline $\mathrm{P}(3)$ & $33(1)$ & $28(1)$ & $47(1)$ & $-13(1)$ & $-19(1)$ & $-3(1)$ \\
\hline $\mathrm{N}(1)$ & $23(2)$ & $18(2)$ & $27(2)$ & $-3(1)$ & $-9(2)$ & $-3(1)$ \\
\hline $\mathrm{N}(2)$ & $24(2)$ & $18(2)$ & $23(2)$ & $-1(1)$ & $-10(1)$ & $-2(1)$ \\
\hline $\mathrm{N}(3)$ & $34(2)$ & $18(2)$ & $33(2)$ & $-7(1)$ & $-19(2)$ & $2(1)$ \\
\hline $\mathrm{O}(1)$ & $39(2)$ & $27(2)$ & $48(2)$ & $-13(1)$ & $-14(2)$ & $8(2)$ \\
\hline $\mathrm{O}(2)$ & $34(2)$ & $27(2)$ & $40(2)$ & $-6(1)$ & $-17(1)$ & $-8(1)$ \\
\hline $\mathrm{O}(3)$ & $29(2)$ & $22(1)$ & $38(2)$ & $-11(1)$ & $-16(1)$ & $4(1)$ \\
\hline$C(1)$ & $26(2)$ & $23(2)$ & $25(2)$ & $-2(2)$ & $-9(2)$ & $-8(2)$ \\
\hline$C(2)$ & $27(2)$ & $19(2)$ & $25(2)$ & $-4(2)$ & $-15(2)$ & $-1(2)$ \\
\hline$C(3)$ & $24(2)$ & $18(2)$ & $22(2)$ & $-4(2)$ & $-8(2)$ & $-4(2)$ \\
\hline$C(4)$ & $20(2)$ & $18(2)$ & $26(2)$ & $-5(2)$ & $-8(2)$ & $-2(2)$ \\
\hline$C(5)$ & $28(2)$ & $17(2)$ & $26(2)$ & $-2(2)$ & $-9(2)$ & $-2(2)$ \\
\hline$C(6)$ & $43(3)$ & $21(2)$ & $33(2)$ & $-8(2)$ & $-21(2)$ & $-1(2)$ \\
\hline$C(7)$ & $42(3)$ & $16(2)$ & $33(2)$ & $-8(2)$ & $-18(2)$ & $2(2)$ \\
\hline$C(8)$ & $76(4)$ & $26(2)$ & $61(3)$ & $-7(2)$ & $-48(3)$ & $-5(2)$ \\
\hline$C(9)$ & $118(5)$ & $27(3)$ & $65(4)$ & $-3(2)$ & $-65(4)$ & $2(3)$ \\
\hline$C(10)$ & $94(5)$ & $27(3)$ & $40(3)$ & $-4(2)$ & $-10(3)$ & $-11(3)$ \\
\hline$C(11)$ & $42(3)$ & $32(3)$ & $81(4)$ & $-16(3)$ & 2(3) & $-4(2)$ \\
\hline$C(12)$ & $40(3)$ & $25(2)$ & $64(3)$ & $-10(2)$ & $-16(2)$ & $7(2)$ \\
\hline$C(13)$ & $26(2)$ & $28(2)$ & $25(2)$ & $-5(2)$ & $-9(2)$ & $-2(2)$ \\
\hline$C(14)$ & $30(2)$ & $33(2)$ & $38(2)$ & $-10(2)$ & $-13(2)$ & $-8(2)$ \\
\hline$C(15)$ & $25(2)$ & $24(2)$ & $38(2)$ & $-5(2)$ & $-4(2)$ & $-9(2)$ \\
\hline$C(16)$ & $23(2)$ & $22(2)$ & $35(2)$ & $-4(2)$ & $-6(2)$ & $-5(2)$ \\
\hline$C(17)$ & $26(2)$ & $21(2)$ & $27(2)$ & $-4(2)$ & $-10(2)$ & $1(2)$ \\
\hline $\mathrm{C}(18)$ & $25(2)$ & $20(2)$ & $25(2)$ & $-3(2)$ & $-8(2)$ & $0(2)$ \\
\hline$C(19)$ & $28(2)$ & $30(2)$ & $25(2)$ & $-3(2)$ & $-7(2)$ & $-1(2)$ \\
\hline$C(20)$ & $42(3)$ & $40(3)$ & $23(2)$ & $-1(2)$ & $-15(2)$ & $-3(2)$ \\
\hline $\mathrm{C}(21)$ & $43(3)$ & $37(3)$ & $36(2)$ & $-7(2)$ & $-24(2)$ & $-2(2)$ \\
\hline$C(22)$ & $28(2)$ & $23(2)$ & $28(2)$ & $-5(2)$ & $-12(2)$ & $-2(2)$ \\
\hline$C(23)$ & $41(3)$ & $32(2)$ & $27(2)$ & $3(2)$ & $-5(2)$ & $-8(2)$ \\
\hline$C(24)$ & $37(3)$ & $26(2)$ & $32(2)$ & $4(2)$ & $-4(2)$ & $-13(2)$ \\
\hline$C(25)$ & $28(2)$ & $18(2)$ & $17(2)$ & $-3(2)$ & $-8(2)$ & $-3(2)$ \\
\hline
\end{tabular}




\begin{tabular}{|c|c|c|c|c|c|c|}
\hline$C(26)$ & $31(2)$ & $20(2)$ & $34(2)$ & $-5(2)$ & $-12(2)$ & $-1(2)$ \\
\hline$C(27)$ & $41(3)$ & $14(2)$ & $39(2)$ & $-3(2)$ & $-11(2)$ & $-3(2)$ \\
\hline $\mathrm{C}(28)$ & $39(3)$ & $26(2)$ & $24(2)$ & $-2(2)$ & $-6(2)$ & $-15(2)$ \\
\hline$C(29)$ & $29(2)$ & $34(2)$ & $27(2)$ & $-7(2)$ & $-9(2)$ & $-2(2)$ \\
\hline$C(30)$ & $30(2)$ & $18(2)$ & $33(2)$ & $-4(2)$ & $-12(2)$ & $0(2)$ \\
\hline$C(31)$ & $28(2)$ & $12(2)$ & $34(2)$ & $-5(2)$ & $-17(2)$ & $2(2)$ \\
\hline$C(32)$ & $34(2)$ & $29(2)$ & $34(2)$ & $-9(2)$ & $-17(2)$ & $3(2)$ \\
\hline$C(33)$ & $56(3)$ & $37(3)$ & $41(3)$ & $-10(2)$ & $-33(3)$ & $7(2)$ \\
\hline$C(34)$ & $43(3)$ & $33(2)$ & $63(3)$ & $-16(2)$ & $-37(3)$ & $10(2)$ \\
\hline$C(35)$ & $27(2)$ & $34(2)$ & $54(3)$ & $-15(2)$ & $-18(2)$ & $3(2)$ \\
\hline$C(36)$ & $30(2)$ & $24(2)$ & $37(2)$ & $-8(2)$ & $-15(2)$ & $-2(2)$ \\
\hline$C(37)$ & $19(2)$ & $15(2)$ & $27(2)$ & $-4(2)$ & $-5(2)$ & $1(2)$ \\
\hline$C(38)$ & $32(2)$ & $24(2)$ & $35(2)$ & $-3(2)$ & $-12(2)$ & $-2(2)$ \\
\hline$C(39)$ & $35(3)$ & $23(2)$ & $48(3)$ & $-5(2)$ & $-10(2)$ & $-7(2)$ \\
\hline$C(40)$ & $44(3)$ & $21(2)$ & $43(3)$ & $10(2)$ & $-6(2)$ & $-2(2)$ \\
\hline$C(41)$ & $47(3)$ & $35(3)$ & $33(2)$ & $3(2)$ & $-14(2)$ & $7(2)$ \\
\hline$C(42)$ & $33(2)$ & $27(2)$ & $31(2)$ & $-1(2)$ & $-14(2)$ & $1(2)$ \\
\hline$C(43)$ & $22(2)$ & $14(2)$ & $35(2)$ & $-4(2)$ & $-13(2)$ & $0(2)$ \\
\hline$C(44)$ & $28(2)$ & $24(2)$ & $32(2)$ & $-5(2)$ & $-15(2)$ & $1(2)$ \\
\hline$C(45)$ & $38(3)$ & $28(2)$ & $44(3)$ & $-9(2)$ & $-25(2)$ & $0(2)$ \\
\hline$C(46)$ & $27(2)$ & $28(2)$ & $59(3)$ & $-13(2)$ & $-21(2)$ & $0(2)$ \\
\hline$C(47)$ & $22(2)$ & $28(2)$ & $42(2)$ & $-8(2)$ & $-8(2)$ & $0(2)$ \\
\hline$C(48)$ & $29(2)$ & $19(2)$ & $27(2)$ & $-8(2)$ & $-9(2)$ & $1(2)$ \\
\hline$C(49)$ & $44(3)$ & $32(2)$ & $43(3)$ & $-12(2)$ & $-17(2)$ & $5(2)$ \\
\hline $\mathrm{Cl}(1)$ & $51(1)$ & $33(1)$ & $47(1)$ & $-12(1)$ & $-21(1)$ & $1(1)$ \\
\hline $\mathrm{Cl}(2)$ & $62(1)$ & $68(1)$ & $80(1)$ & $-42(1)$ & $-43(1)$ & $13(1)$ \\
\hline $\mathrm{Cl}(3)$ & $95(1)$ & $36(1)$ & $90(1)$ & 4(1) & $-58(1)$ & $6(1)$ \\
\hline $\mathrm{F}(1)$ & $133(4)$ & $73(2)$ & $84(3)$ & $-56(2)$ & $-42(3)$ & $15(2)$ \\
\hline $\mathrm{F}(2)$ & $47(2)$ & $58(2)$ & $62(2)$ & $-31(2)$ & $-22(2)$ & $20(1)$ \\
\hline $\mathrm{F}(3)$ & $62(2)$ & $58(2)$ & $153(4)$ & $-10(2)$ & $-51(2)$ & $-27(2)$ \\
\hline $\mathrm{F}(4)$ & $85(2)$ & $73(2)$ & $72(2)$ & $-7(2)$ & $-55(2)$ & $3(2)$ \\
\hline$F(5)$ & $34(2)$ & $46(2)$ & $80(2)$ & $-12(2)$ & $-15(2)$ & $-10(1)$ \\
\hline $\mathrm{F}(6)$ & $56(2)$ & $52(2)$ & $56(2)$ & $-4(2)$ & $-20(2)$ & $18(2)$ \\
\hline
\end{tabular}

The anisotropic displacement factor exponent takes the form: $-2 \pi^{2}\left[\mathrm{~h}^{2} \mathrm{a}^{* 2} \mathrm{U}_{11}+\ldots+\right.$ $\left.2 \mathrm{hka} * \mathrm{~b} * \mathrm{U}_{12}\right]$ 
Table S6. Hydrogen coordinates $\left(\times 10^{4}\right)$ and isotropic displacement parameters $\left(\AA^{2} \times 10^{3}\right)$ for $\left.\left[\mathrm{Re}(\mathrm{phen})(\mathrm{PP}-\mathrm{Bn})(\mathrm{CO})_{2}\right]\left(\mathrm{PF}_{6}\right) \cdot \mathrm{CDCl}_{3}\right]$.

\begin{tabular}{|c|c|c|c|c|}
\hline Atom & $\mathrm{x}$ & $\mathrm{y}$ & $\mathrm{z}$ & $\mathrm{U}(\mathrm{eq})$ \\
\hline $\mathrm{H}(5)$ & 6480 & 8173 & 8429 & 32 \\
\hline $\mathrm{H}(3)$ & 6203 & 6538 & 8615 & 26 \\
\hline $\mathrm{H}(6 \mathrm{~A})$ & 5821 & 9294 & 9353 & 37 \\
\hline $\mathrm{H}(6 \mathrm{~B})$ & 4720 & 9360 & 9183 & 37 \\
\hline $\mathrm{H}(8)$ & 4557 & 10650 & 7802 & 57 \\
\hline $\mathrm{H}(9)$ & 5200 & 12164 & 6584 & 74 \\
\hline $\mathrm{H}(10)$ & 6961 & 12785 & 6142 & 73 \\
\hline $\mathrm{H}(11)$ & 8069 & 11914 & 6931 & 73 \\
\hline $\mathrm{H}(12)$ & 7446 & 10383 & 8113 & 55 \\
\hline $\mathrm{H}(13)$ & 8806 & 7102 & 6251 & 33 \\
\hline $\mathrm{H}(14)$ & 9987 & 8482 & 5533 & 40 \\
\hline $\mathrm{H}(15)$ & 10387 & 9399 & 3892 & 39 \\
\hline $\mathrm{H}(20)$ & 8094 & 7778 & 1396 & 43 \\
\hline $\mathrm{H}(21)$ & 6847 & 6456 & 2312 & 44 \\
\hline $\mathrm{H}(22)$ & 6465 & 5725 & 3993 & 31 \\
\hline $\mathrm{H}(23)$ & 9423 & 9012 & 1394 & 46 \\
\hline $\mathrm{H}(24)$ & 10162 & 9582 & 2256 & 44 \\
\hline $\mathrm{H}(26)$ & 6863 & 3365 & 8156 & 35 \\
\hline $\mathrm{H}(27)$ & 5531 & 2096 & 8989 & 42 \\
\hline $\mathrm{H}(28)$ & 3629 & 2464 & 9599 & 39 \\
\hline $\mathrm{H}(29)$ & 3071 & 4118 & 9377 & 38 \\
\hline $\mathrm{H}(30)$ & 4410 & 5394 & 8503 & 34 \\
\hline $\mathrm{H}(32)$ & 6997 & 4888 & 9353 & 38 \\
\hline $\mathrm{H}(33)$ & 8425 & 4411 & 9883 & 49 \\
\hline $\mathrm{H}(34)$ & 10241 & 4264 & 8721 & 50 \\
\hline $\mathrm{H}(35)$ & 10668 & 4650 & 7013 & 45 \\
\hline $\mathrm{H}(36)$ & 9261 & 5199 & 6450 & 36 \\
\hline $\mathrm{H}(38)$ & 7066 & 8969 & 6150 & 38 \\
\hline $\mathrm{H}(39)$ & 7681 & 10510 & 5008 & 46 \\
\hline $\mathrm{H}(40)$ & 7235 & 11033 & 3632 & 53 \\
\hline $\mathrm{H}(41)$ & 6139 & 10017 & 3412 & 51 \\
\hline $\mathrm{H}(42)$ & 5505 & 8476 & 4538 & 38 \\
\hline $\mathrm{H}(44)$ & 4810 & 6691 & 5224 & 34 \\
\hline $\mathrm{H}(45)$ & 3018 & 6327 & 5492 & 41 \\
\hline $\mathrm{H}(46)$ & 1485 & 6398 & 7010 & 44 \\
\hline
\end{tabular}




\begin{tabular}{rrrrr}
$\mathrm{H}(47)$ & 1782 & 6834 & 8250 & 39 \\
$\mathrm{H}(48)$ & 3573 & 7244 & 7973 & 31 \\
$\mathrm{H}(49)$ & 7148 & 997 & 1207 & 47 \\
\hline
\end{tabular}




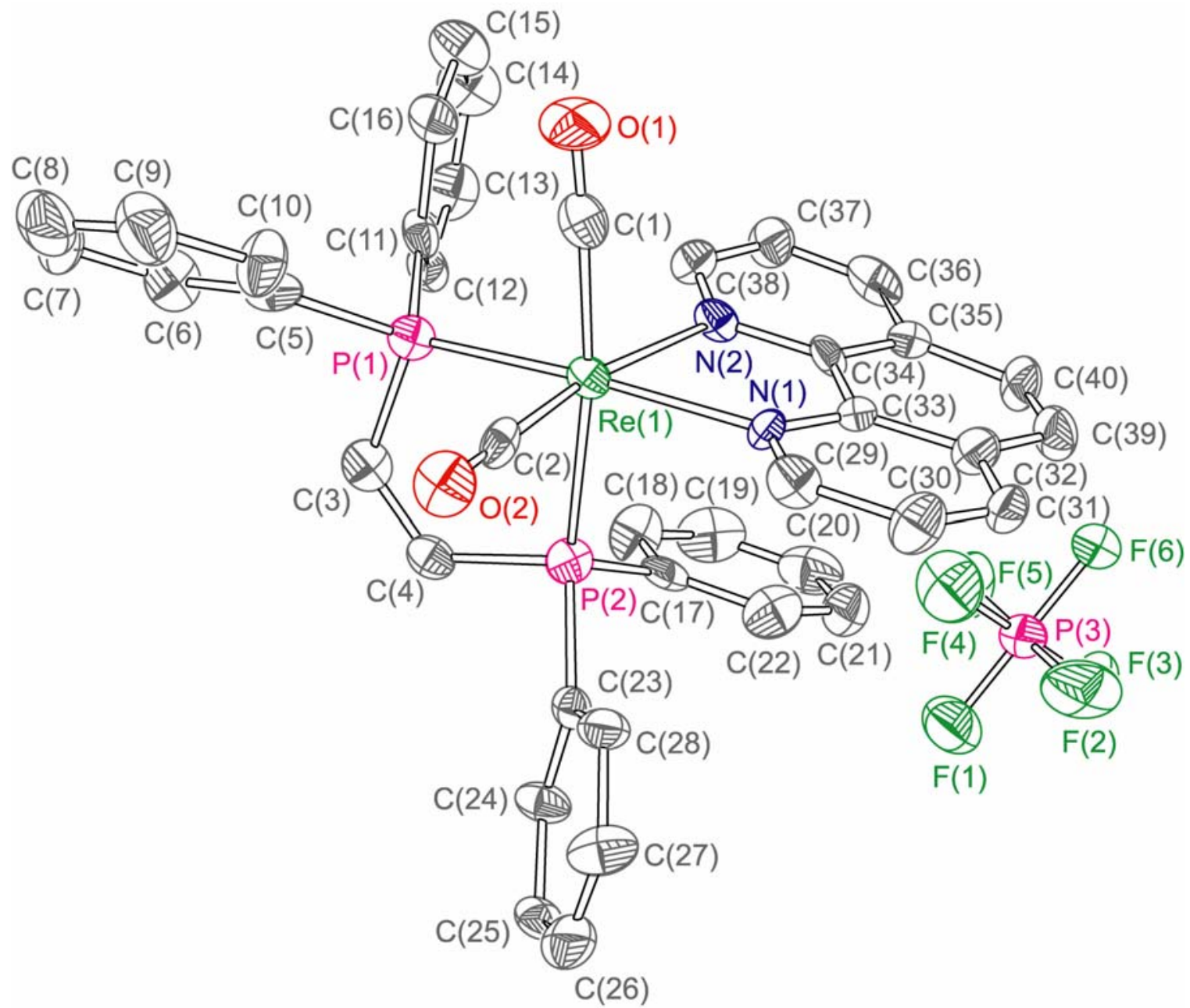

Figure S2. Atom labeling scheme for $\left[\mathrm{Re}(\mathrm{phen})(\mathrm{dppe})(\mathrm{CO})_{2}\right]\left(\mathrm{PF}_{6}\right)$. 
Table S7. Crystal data and structure refinement for $\left[\mathrm{Re}(\right.$ phen $\left.)(\mathrm{dppe})(\mathrm{CO})_{2}\right]\left(\mathrm{PF}_{6}\right)$.

\begin{tabular}{|c|c|}
\hline Empirical formula & $\mathrm{C}_{40} \mathrm{H}_{30} \mathrm{~F}_{6} \mathrm{~N}_{2} \mathrm{O}_{2} \mathrm{P}_{3} \mathrm{Re}$ \\
\hline Formula weight & 963.77 \\
\hline $\mathrm{T}(\mathrm{K})$ & $150(2)$ \\
\hline$\lambda(\AA)$ & 0.71073 \\
\hline Crystal system & Monoclinic \\
\hline Space group & $P 2(1) / \mathrm{n}$ \\
\hline$a(\AA)$ & $15.1299(10)$ \\
\hline$b(\AA)$ & $15.6153(11)$ \\
\hline$c(\AA)$ & $16.7302(12)$ \\
\hline$\alpha\left(^{\circ}\right)$ & 90 \\
\hline$\beta\left(^{\circ}\right)$ & $109.328(2)$ \\
\hline$\gamma\left(\left(^{\circ}\right)\right.$ & 90 \\
\hline$V\left(\AA^{3}\right)$ & $3729.9(4)$ \\
\hline$Z$ & 4 \\
\hline$\rho_{\text {calcd }}\left(\mathrm{Mg} / \mathrm{m}^{3}\right)$ & 1.716 \\
\hline Abs. coeff $\left(\mathrm{mm}^{-1}\right)$ & 3.456 \\
\hline$\theta$ range for data collection $\left({ }^{\circ}\right)$ & 1.83 to 23.15 \\
\hline Index ranges & $-16 \leq h \leq 15,0 \leq k \leq 17,0 \leq \ell \leq 18$ \\
\hline Reflections collected & 5287 \\
\hline Independent reflns & 5287 \\
\hline Completeness to $\theta_{\max }$ & $99.8 \%$ \\
\hline Data/restraints/parameters & $5287 / 0 / 487$ \\
\hline $\mathrm{GOF}^{a}$ on $F^{2}$ & 0.970 \\
\hline $\mathrm{R} 1^{b}$ & 0.0546 \\
\hline $\mathrm{wR} 2^{c}$ & 0.0981 \\
\hline Largest diff. peak, hole & 1.074 and -0.672 e. $\AA^{-3}$ \\
\hline
\end{tabular}

${ }^{\mathrm{a}} \mathrm{GOF}=\left(\Sigma w\left(F_{\mathrm{o}}{ }^{2}-F_{\mathrm{c}}{ }^{2}\right)^{2} /(n-p)\right)^{1 / 2}$ where $n$ is the number of data and $p$ is the number of parameters refined. ${ }^{b} R 1=\Sigma|| F_{\mathrm{o}}-\left|F_{\mathrm{c}}\right||\Sigma| F_{\mathrm{o}} \mid .{ }^{c} \mathrm{w} R 2=\left(\Sigma\left(w\left(F_{\mathrm{o}}{ }^{2}-F_{\mathrm{c}}{ }^{2}\right)^{2}\right) / \Sigma\left(w\left(F_{\mathrm{o}}{ }^{2}\right)^{2}\right)\right)^{1 / 2}$. 
Table S8. Atomic coordinates $\left(\times 10^{4}\right)$ and equivalent isotropic displacement parameters $\left(\AA^{2}\right.$ $\times 10^{3}$ ) for $\left[\operatorname{Re}(\right.$ phen $)($ dppe $\left.)(\mathrm{CO})_{2}\right]\left(\mathrm{PF}_{6}\right)$.

\begin{tabular}{|c|c|c|c|c|}
\hline Atom & $\mathrm{x}$ & $\mathrm{y}$ & z & $\mathrm{U}_{\mathrm{eq}}$ \\
\hline $\operatorname{Re}(1)$ & $6014(1)$ & 1661(1) & $9465(1)$ & $23(1)$ \\
\hline $\mathrm{P}(1)$ & $7280(2)$ & $1247(2)$ & $9026(2)$ & $27(1)$ \\
\hline $\mathrm{P}(2)$ & 6991(2) & $2939(2)$ & $9930(2)$ & $28(1)$ \\
\hline $\mathrm{P}(3)$ & $4812(2)$ & $8227(2)$ & $6668(2)$ & $41(1)$ \\
\hline $\mathrm{F}(1)$ & $5806(5)$ & $8515(5)$ & $7277(5)$ & $77(3)$ \\
\hline $\mathrm{F}(2)$ & $4426(6)$ & $8187(7)$ & $7437(5)$ & $94(3)$ \\
\hline $\mathrm{F}(3)$ & $4500(5)$ & $9204(5)$ & $6500(5)$ & $73(3)$ \\
\hline $\mathrm{F}(4)$ & $5185(5)$ & $8278(6)$ & $5888(4)$ & $75(3)$ \\
\hline $\mathrm{F}(5)$ & $5124(6)$ & $7260(5)$ & $6824(6)$ & $86(3)$ \\
\hline $\mathrm{F}(6)$ & $3805(4)$ & $7956(5)$ & $6038(4)$ & $54(2)$ \\
\hline $\mathrm{N}(1)$ & $4882(6)$ & $2207(6)$ & $9832(6)$ & $24(2)$ \\
\hline $\mathrm{N}(2)$ & $5138(6)$ & $2399(6)$ & $8339(5)$ & $25(2)$ \\
\hline $\mathrm{O}(1)$ & $5047(6)$ & $-55(6)$ & $8837(5)$ & $48(2)$ \\
\hline $\mathrm{O}(2)$ & $6906(5)$ & $781(5)$ & $11173(5)$ & $40(2)$ \\
\hline $\mathrm{C}(1)$ & $5387(8)$ & $595(9)$ & $9066(7)$ & $35(3)$ \\
\hline$C(2)$ & $6586(7)$ & $1123(7)$ & $10522(8)$ & $26(3)$ \\
\hline$C(3)$ & $8221(8)$ & $2026(8)$ & $9399(7)$ & $33(3)$ \\
\hline$C(4)$ & $8107(7)$ & $2728(8)$ & $9781(7)$ & $29(3)$ \\
\hline$C(5)$ & $7890(8)$ & $231(8)$ & $9367(7)$ & $34(3)$ \\
\hline$C(6)$ & $8697(8)$ & $61(9)$ & $9137(7)$ & $40(3)$ \\
\hline$C(7)$ & $9194(8)$ & $-692(10)$ & $9382(9)$ & $50(4)$ \\
\hline $\mathrm{C}(8)$ & $8897(10)$ & $-1256(9)$ & $9860(9)$ & $56(4)$ \\
\hline $\mathrm{C}(9)$ & $8101(9)$ & $-1123(9)$ & $10071(9)$ & $55(4)$ \\
\hline$C(10)$ & $7596(8)$ & $-384(8)$ & $9820(8)$ & $40(3)$ \\
\hline $\mathrm{C}(11)$ & $7074(7)$ & $1221(7)$ & $7893(7)$ & $28(3)$ \\
\hline$C(12)$ & $7381(7)$ & $1882(8)$ & $7471(7)$ & $33(3)$ \\
\hline$C(13)$ & $7068(9)$ & 1895(9) & $6587(8)$ & $45(4)$ \\
\hline$C(14)$ & $6472(9)$ & $1284(9)$ & $6134(8)$ & $49(4)$ \\
\hline$C(15)$ & $6204(8)$ & $635(9)$ & $6540(7)$ & $44(4)$ \\
\hline$C(16)$ & $6504(7)$ & $576(8)$ & $7417(7)$ & $38(3)$ \\
\hline$C(17)$ & $6585(7)$ & $3909(8)$ & $9294(7)$ & $28(3)$ \\
\hline $\mathrm{C}(18)$ & $6854(8)$ & 4097(9) & $8611(8)$ & $43(3)$ \\
\hline C(19) & $6446(10)$ & 4799(9) & $8086(8)$ & $54(4)$ \\
\hline$C(20)$ & $5786(10)$ & $5279(9)$ & $8236(8)$ & $52(4)$ \\
\hline $\mathrm{C}(21)$ & $5509(8)$ & $5093(8)$ & $8921(8)$ & $43(3)$ \\
\hline
\end{tabular}




\begin{tabular}{lllll}
$\mathrm{C}(22)$ & $5912(9)$ & $4400(8)$ & $9452(8)$ & $42(3)$ \\
$\mathrm{C}(23)$ & $7345(7)$ & $3324(8)$ & $11019(6)$ & $23(3)$ \\
$\mathrm{C}(24)$ & $8014(7)$ & $3971(7)$ & $11292(7)$ & $31(3)$ \\
$\mathrm{C}(25)$ & $8325(7)$ & $4250(7)$ & $12128(7)$ & $29(3)$ \\
$\mathrm{C}(26)$ & $7928(8)$ & $3893(8)$ & $12672(8)$ & $42(3)$ \\
$\mathrm{C}(27)$ & $7250(8)$ & $3270(8)$ & $12435(7)$ & $39(3)$ \\
$\mathrm{C}(28)$ & $6957(7)$ & $2991(7)$ & $11589(7)$ & $31(3)$ \\
$\mathrm{C}(29)$ & $4726(7)$ & $2070(8)$ & $10553(7)$ & $32(3)$ \\
$\mathrm{C}(30)$ & $4018(8)$ & $2488(8)$ & $10771(7)$ & $39(3)$ \\
$\mathrm{C}(31)$ & $3478(7)$ & $3085(7)$ & $10222(7)$ & $31(3)$ \\
$\mathrm{C}(32)$ & $3606(7)$ & $3234(8)$ & $9443(7)$ & $30(3)$ \\
$\mathrm{C}(33)$ & $4319(7)$ & $2774(7)$ & $9278(7)$ & $26(3)$ \\
$\mathrm{C}(34)$ & $4451(7)$ & $2883(7)$ & $8478(7)$ & $23(3)$ \\
$\mathrm{C}(35)$ & $3909(7)$ & $3458(8)$ & $7870(7)$ & $28(3)$ \\
$\mathrm{C}(36)$ & $4052(8)$ & $3485(8)$ & $7080(7)$ & $37(3)$ \\
$\mathrm{C}(37)$ & $4691(8)$ & $2960(8)$ & $6942(7)$ & $36(3)$ \\
$\mathrm{C}(38)$ & $5222(8)$ & $2421(7)$ & $7585(7)$ & $31(3)$ \\
$\mathrm{C}(39)$ & $3081(8)$ & $3829(8)$ & $8827(7)$ & $37(3)$ \\
$\mathrm{C}(40)$ & $3238(8)$ & $3958(8)$ & $8088(7)$ & $36(3)$ \\
\hline
\end{tabular}

${ }^{a} \mathrm{U}_{\mathrm{eq}}$ is defined as one third of the trace of the orthogonalized $\mathrm{U}_{\mathrm{ij}}$ tensor. 
Table S9. Bond lengths $(\AA)$ for $\left[\mathrm{Re}(\right.$ phen $\left.)(\mathrm{dppe})(\mathrm{CO})_{2}\right]\left(\mathrm{PF}_{6}\right)$.

\begin{tabular}{|c|c|c|c|}
\hline Bond & Length & Bond & Length \\
\hline $\operatorname{Re}(1)-C(2)$ & $1.889(13)$ & $C(11)-C(16)$ & $1.392(15)$ \\
\hline $\operatorname{Re}(1)-C(1)$ & $1.921(14)$ & $\mathrm{C}(11)-\mathrm{C}(12)$ & $1.413(15)$ \\
\hline $\operatorname{Re}(1)-\mathrm{N}(1)$ & $2.175(8)$ & $\mathrm{C}(12)-\mathrm{C}(13)$ & $1.396(15)$ \\
\hline $\operatorname{Re}(1)-\mathrm{N}(2)$ & $2.232(8)$ & $\mathrm{C}(13)-\mathrm{C}(14)$ & $1.358(16)$ \\
\hline $\operatorname{Re}(1)-\mathrm{P}(1)$ & $2.358(3)$ & $C(14)-C(15)$ & $1.354(16)$ \\
\hline $\operatorname{Re}(1)-\mathrm{P}(2)$ & $2.454(3)$ & $C(15)-C(16)$ & $1.388(14)$ \\
\hline $\mathrm{P}(1)-\mathrm{C}(11)$ & $1.817(12)$ & $\mathrm{C}(17)-\mathrm{C}(18)$ & $1.367(15)$ \\
\hline $\mathrm{P}(1)-\mathrm{C}(3)$ & $1.819(12)$ & $\mathrm{C}(17)-\mathrm{C}(22)$ & $1.368(15)$ \\
\hline $\mathrm{P}(1)-\mathrm{C}(5)$ & $1.830(12)$ & $\mathrm{C}(18)-\mathrm{C}(19)$ & $1.413(17)$ \\
\hline$P(2)-C(4)$ & $1.817(11)$ & $C(19)-C(20)$ & $1.337(17)$ \\
\hline $\mathrm{P}(2)-\mathrm{C}(23)$ & $1.824(10)$ & $\mathrm{C}(20)-\mathrm{C}(21)$ & $1.376(16)$ \\
\hline $\mathrm{P}(2)-\mathrm{C}(17)$ & $1.835(12)$ & $\mathrm{C}(21)-\mathrm{C}(22)$ & $1.405(16)$ \\
\hline $\mathrm{P}(3)-\mathrm{F}(1)$ & $1.578(8)$ & $\mathrm{C}(23)-\mathrm{C}(28)$ & $1.377(14)$ \\
\hline $\mathrm{P}(3)-\mathrm{F}(5)$ & $1.579(9)$ & $\mathrm{C}(23)-\mathrm{C}(24)$ & $1.394(14)$ \\
\hline$P(3)-F(2)$ & $1.580(8)$ & $C(24)-C(25)$ & $1.390(14)$ \\
\hline$P(3)-F(4)$ & $1.585(7)$ & $C(25)-C(26)$ & $1.365(15)$ \\
\hline $\mathrm{P}(3)-\mathrm{F}(3)$ & $1.594(8)$ & $C(26)-C(27)$ & $1.374(16)$ \\
\hline$P(3)-F(6)$ & $1.595(7)$ & $\mathrm{C}(27)-\mathrm{C}(28)$ & $1.405(14)$ \\
\hline $\mathrm{N}(1)-\mathrm{C}(29)$ & $1.321(13)$ & $\mathrm{C}(29)-\mathrm{C}(30)$ & $1.402(14)$ \\
\hline $\mathrm{N}(1)-\mathrm{C}(33)$ & $1.358(13)$ & $\mathrm{C}(30)-\mathrm{C}(31)$ & $1.372(15)$ \\
\hline $\mathrm{N}(2)-\mathrm{C}(38)$ & $1.309(13)$ & $\mathrm{C}(31)-\mathrm{C}(32)$ & $1.399(14)$ \\
\hline $\mathrm{N}(2)-\mathrm{C}(34)$ & $1.366(12)$ & $\mathrm{C}(32)-\mathrm{C}(33)$ & $1.397(14)$ \\
\hline $\mathrm{O}(1)-\mathrm{C}(1)$ & $1.145(13)$ & $C(32)-C(39)$ & $1.420(15)$ \\
\hline $\mathrm{O}(2)-\mathrm{C}(2)$ & $1.165(12)$ & $\mathrm{C}(33)-\mathrm{C}(34)$ & $1.428(14)$ \\
\hline$C(3)-C(4)$ & $1.309(15)$ & $C(34)-C(35)$ & $1.401(14)$ \\
\hline$C(5)-C(10)$ & $1.384(15)$ & $C(35)-C(36)$ & $1.408(14)$ \\
\hline$C(5)-C(6)$ & $1.421(14)$ & $C(35)-C(40)$ & $1.420(14)$ \\
\hline$C(6)-C(7)$ & $1.383(17)$ & $C(36)-C(37)$ & $1.345(15)$ \\
\hline$C(7)-C(8)$ & $1.360(18)$ & $\mathrm{C}(37)-\mathrm{C}(38)$ & $1.393(14)$ \\
\hline$C(8)-C(9)$ & $1.379(18)$ & $\mathrm{C}(39)-\mathrm{C}(40)$ & $1.350(15)$ \\
\hline $\mathrm{C}(9)-\mathrm{C}(10)$ & $1.371(16)$ & & \\
\hline
\end{tabular}


Table S10. Bond angles $\left(^{\circ}\right)$ for $\left[\operatorname{Re}(\right.$ phen $\left.)(\mathrm{dppe})(\mathrm{CO})_{2}\right]\left(\mathrm{PF}_{6}\right)$.

\begin{tabular}{|c|c|c|c|}
\hline Bond angle & Angle & Bond angle & Angle \\
\hline $\mathrm{C}(2)-\operatorname{Re}(1)-\mathrm{C}(1)$ & $87.8(5)$ & $C(10)-C(5)-C(6)$ & $118.6(11)$ \\
\hline $\mathrm{C}(2)-\operatorname{Re}(1)-\mathrm{N}(1)$ & $94.0(4)$ & $\mathrm{C}(10)-\mathrm{C}(5)-\mathrm{P}(1)$ & $123.5(9)$ \\
\hline $\mathrm{C}(1)-\operatorname{Re}(1)-\mathrm{N}(1)$ & $95.4(4)$ & $\mathrm{C}(6)-\mathrm{C}(5)-\mathrm{P}(1)$ & $117.9(10)$ \\
\hline $\mathrm{C}(2)-\operatorname{Re}(1)-\mathrm{N}(2)$ & $168.5(4)$ & $C(7)-C(6)-C(5)$ & $120.7(12)$ \\
\hline $\mathrm{C}(1)-\operatorname{Re}(1)-\mathrm{N}(2)$ & $93.9(4)$ & $C(8)-C(7)-C(6)$ & $118.1(12)$ \\
\hline $\mathrm{N}(1)-\operatorname{Re}(1)-\mathrm{N}(2)$ & $74.5(3)$ & $\mathrm{C}(7)-\mathrm{C}(8)-\mathrm{C}(9)$ & $122.7(14)$ \\
\hline $\mathrm{C}(2)-\operatorname{Re}(1)-\mathrm{P}(1)$ & $90.1(3)$ & $C(10)-C(9)-C(8)$ & $119.5(14)$ \\
\hline $\mathrm{C}(1)-\operatorname{Re}(1)-\mathrm{P}(1)$ & $90.8(4)$ & $C(9)-C(10)-C(5)$ & $120.3(12)$ \\
\hline $\mathrm{N}(1)-\operatorname{Re}(1)-\mathrm{P}(1)$ & $172.7(3)$ & $\mathrm{C}(16)-\mathrm{C}(11)-\mathrm{C}(12)$ & $119.2(11)$ \\
\hline $\mathrm{N}(2)-\operatorname{Re}(1)-\mathrm{P}(1)$ & $101.2(2)$ & $\mathrm{C}(16)-\mathrm{C}(11)-\mathrm{P}(1)$ & $118.2(9)$ \\
\hline $\mathrm{C}(2)-\mathrm{Re}(1)-\mathrm{P}(2)$ & 91.3(3) & $\mathrm{C}(12)-\mathrm{C}(11)-\mathrm{P}(1)$ & $122.2(9)$ \\
\hline $\mathrm{C}(1)-\operatorname{Re}(1)-\mathrm{P}(2)$ & $172.3(4)$ & $\mathrm{C}(13)-\mathrm{C}(12)-\mathrm{C}(11)$ & $119.1(11)$ \\
\hline $\mathrm{N}(1)-\operatorname{Re}(1)-\mathrm{P}(2)$ & $92.3(2)$ & $\mathrm{C}(14)-\mathrm{C}(13)-\mathrm{C}(12)$ & $120.7(12)$ \\
\hline $\mathrm{N}(2)-\mathrm{Re}(1)-\mathrm{P}(2)$ & $88.5(2)$ & $\mathrm{C}(15)-\mathrm{C}(14)-\mathrm{C}(13)$ & $120.0(12)$ \\
\hline $\mathrm{P}(1)-\mathrm{Re}(1)-\mathrm{P}(2)$ & $81.63(10)$ & $\mathrm{C}(14)-\mathrm{C}(15)-\mathrm{C}(16)$ & $122.2(13)$ \\
\hline $\mathrm{C}(11)-\mathrm{P}(1)-\mathrm{C}(3)$ & $102.6(5)$ & $C(15)-C(16)-C(11)$ & $118.7(12)$ \\
\hline $\mathrm{C}(11)-\mathrm{P}(1)-\mathrm{C}(5)$ & $101.6(5)$ & $\mathrm{C}(18)-\mathrm{C}(17)-\mathrm{C}(22)$ & $119.2(12)$ \\
\hline $\mathrm{C}(3)-\mathrm{P}(1)-\mathrm{C}(5)$ & $102.4(5)$ & $\mathrm{C}(18)-\mathrm{C}(17)-\mathrm{P}(2)$ & $121.7(10)$ \\
\hline $\mathrm{C}(11)-\mathrm{P}(1)-\operatorname{Re}(1)$ & 117.1(3) & $\mathrm{C}(22)-\mathrm{C}(17)-\mathrm{P}(2)$ & $118.6(9)$ \\
\hline $\mathrm{C}(3)-\mathrm{P}(1)-\operatorname{Re}(1)$ & $109.6(4)$ & $\mathrm{C}(17)-\mathrm{C}(18)-\mathrm{C}(19)$ & $119.7(12)$ \\
\hline $\mathrm{C}(5)-\mathrm{P}(1)-\operatorname{Re}(1)$ & $121.2(4)$ & $\mathrm{C}(20)-\mathrm{C}(19)-\mathrm{C}(18)$ & $121.2(13)$ \\
\hline $\mathrm{C}(4)-\mathrm{P}(2)-\mathrm{C}(23)$ & $102.6(5)$ & $\mathrm{C}(19)-\mathrm{C}(20)-\mathrm{C}(21)$ & $119.4(14)$ \\
\hline $\mathrm{C}(4)-\mathrm{P}(2)-\mathrm{C}(17)$ & $102.6(5)$ & $\mathrm{C}(20)-\mathrm{C}(21)-\mathrm{C}(22)$ & $120.0(13)$ \\
\hline $\mathrm{C}(23)-\mathrm{P}(2)-\mathrm{C}(17)$ & $103.8(5)$ & $\mathrm{C}(17)-\mathrm{C}(22)-\mathrm{C}(21)$ & $120.3(12)$ \\
\hline $\mathrm{C}(4)-\mathrm{P}(2)-\operatorname{Re}(1)$ & $107.4(4)$ & $\mathrm{C}(28)-\mathrm{C}(23)-\mathrm{C}(24)$ & $118.5(10)$ \\
\hline $\mathrm{C}(23)-\mathrm{P}(2)-\operatorname{Re}(1)$ & $122.1(4)$ & $\mathrm{C}(28)-\mathrm{C}(23)-\mathrm{P}(2)$ & $121.1(9)$ \\
\hline $\mathrm{C}(17)-\mathrm{P}(2)-\operatorname{Re}(1)$ & $115.9(3)$ & $\mathrm{C}(24)-\mathrm{C}(23)-\mathrm{P}(2)$ & $120.4(8)$ \\
\hline $\mathrm{F}(1)-\mathrm{P}(3)-\mathrm{F}(5)$ & $89.8(5)$ & $\mathrm{C}(25)-\mathrm{C}(24)-\mathrm{C}(23)$ & $121.5(11)$ \\
\hline $\mathrm{F}(1)-\mathrm{P}(3)-\mathrm{F}(2)$ & $90.9(5)$ & $\mathrm{C}(26)-\mathrm{C}(25)-\mathrm{C}(24)$ & $117.8(11)$ \\
\hline $\mathrm{F}(5)-\mathrm{P}(3)-\mathrm{F}(2)$ & $89.9(5)$ & $\mathrm{C}(25)-\mathrm{C}(26)-\mathrm{C}(27)$ & $123.2(12)$ \\
\hline $\mathrm{F}(1)-\mathrm{P}(3)-\mathrm{F}(4)$ & $89.6(4)$ & $\mathrm{C}(26)-\mathrm{C}(27)-\mathrm{C}(28)$ & $117.9(12)$ \\
\hline $\mathrm{F}(5)-\mathrm{P}(3)-\mathrm{F}(4)$ & $90.9(5)$ & $\mathrm{C}(23)-\mathrm{C}(28)-\mathrm{C}(27)$ & $121.0(11)$ \\
\hline $\mathrm{F}(2)-\mathrm{P}(3)-\mathrm{F}(4)$ & $179.0(5)$ & $\mathrm{N}(1)-\mathrm{C}(29)-\mathrm{C}(30)$ & $123.0(11)$ \\
\hline $\mathrm{F}(1)-\mathrm{P}(3)-\mathrm{F}(3)$ & $90.2(5)$ & $\mathrm{C}(31)-\mathrm{C}(30)-\mathrm{C}(29)$ & $118.6(11)$ \\
\hline $\mathrm{F}(5)-\mathrm{P}(3)-\mathrm{F}(3)$ & $179.3(5)$ & $\mathrm{C}(30)-\mathrm{C}(31)-\mathrm{C}(32)$ & $120.0(11)$ \\
\hline $\mathrm{F}(2)-\mathrm{P}(3)-\mathrm{F}(3)$ & $90.8(5)$ & $\mathrm{C}(33)-\mathrm{C}(32)-\mathrm{C}(31)$ & $116.9(11)$ \\
\hline
\end{tabular}




\begin{tabular}{lrll}
$\mathrm{F}(4)-\mathrm{P}(3)-\mathrm{F}(3)$ & $88.4(5)$ & $\mathrm{C}(33)-\mathrm{C}(32)-\mathrm{C}(39)$ & $118.5(10)$ \\
$\mathrm{F}(1)-\mathrm{P}(3)-\mathrm{F}(6)$ & $178.6(5)$ & $\mathrm{C}(31)-\mathrm{C}(32)-\mathrm{C}(39)$ & $124.6(11)$ \\
$\mathrm{F}(5)-\mathrm{P}(3)-\mathrm{F}(6)$ & $91.4(4)$ & $\mathrm{N}(1)-\mathrm{C}(33)-\mathrm{C}(32)$ & $123.4(10)$ \\
$\mathrm{F}(2)-\mathrm{P}(3)-\mathrm{F}(6)$ & $89.9(4)$ & $\mathrm{N}(1)-\mathrm{C}(33)-\mathrm{C}(34)$ & $117.8(10)$ \\
$\mathrm{F}(4)-\mathrm{P}(3)-\mathrm{F}(6)$ & $89.5(4)$ & $\mathrm{C}(32)-\mathrm{C}(33)-\mathrm{C}(34)$ & $118.7(10)$ \\
$\mathrm{F}(3)-\mathrm{P}(3)-\mathrm{F}(6)$ & $88.6(4)$ & $\mathrm{N}(2)-\mathrm{C}(34)-\mathrm{C}(35)$ & $121.8(10)$ \\
$\mathrm{C}(29)-\mathrm{N}(1)-\mathrm{C}(33)$ & $117.9(9)$ & $\mathrm{N}(2)-\mathrm{C}(34)-\mathrm{C}(33)$ & $116.4(10)$ \\
$\mathrm{C}(29)-\mathrm{N}(1)-\mathrm{Re}(1)$ & $126.1(8)$ & $\mathrm{C}(35)-\mathrm{C}(34)-\mathrm{C}(33)$ & $121.9(10)$ \\
$\mathrm{C}(33)-\mathrm{N}(1)-\mathrm{Re}(1)$ & $116.0(7)$ & $\mathrm{C}(34)-\mathrm{C}(35)-\mathrm{C}(36)$ & $117.7(10)$ \\
$\mathrm{C}(38)-\mathrm{N}(2)-\mathrm{C}(34)$ & $118.2(9)$ & $\mathrm{C}(34)-\mathrm{C}(35)-\mathrm{C}(40)$ & $117.5(10)$ \\
$\mathrm{C}(38)-\mathrm{N}(2)-\mathrm{Re}(1)$ & $127.3(8)$ & $\mathrm{C}(36)-\mathrm{C}(35)-\mathrm{C}(40)$ & $124.8(11)$ \\
$\mathrm{C}(34)-\mathrm{N}(2)-\mathrm{Re}(1)$ & $114.5(7)$ & $\mathrm{C}(37)-\mathrm{C}(36)-\mathrm{C}(35)$ & $119.1(11)$ \\
$\mathrm{O}(1)-\mathrm{C}(1)-\mathrm{Re}(1)$ & $177.3(10)$ & $\mathrm{C}(36)-\mathrm{C}(37)-\mathrm{C}(38)$ & $120.0(11)$ \\
$\mathrm{O}(2)-\mathrm{C}(2)-\mathrm{Re}(1)$ & $177.4(9)$ & $\mathrm{N}(2)-\mathrm{C}(38)-\mathrm{C}(37)$ & $123.0(11)$ \\
$\mathrm{C}(4)-\mathrm{C}(3)-\mathrm{P}(1)$ & $120.9(9)$ & $\mathrm{C}(40)-\mathrm{C}(39)-\mathrm{C}(32)$ & $122.4(11)$ \\
$\mathrm{C}(3)-\mathrm{C}(4)-\mathrm{P}(2)$ & $119.8(9)$ & $\mathrm{C}(39)-\mathrm{C}(40)-\mathrm{C}(35)$ & $120.8(11)$ \\
\hline
\end{tabular}


Table S11. Anisotropic displacement parameters $\left(\begin{array}{llll}\AA^{2} & \times & 10^{3}\end{array}\right)$ for $\left[\operatorname{Re}(\right.$ phen $)($ dppe $\left.)(\mathrm{CO})_{2}\right]\left(\mathrm{PF}_{6}\right)$.

\begin{tabular}{|c|c|c|c|c|c|c|}
\hline Atom & $\mathrm{U}_{11}$ & $\mathrm{U}_{22}$ & $\mathrm{U}_{33}$ & $\mathrm{U}_{23}$ & $\mathrm{U}_{13}$ & $\mathrm{U}_{12}$ \\
\hline $\operatorname{Re}(1)$ & $22(1)$ & $25(1)$ & $22(1)$ & $2(1)$ & $6(1)$ & $-2(1)$ \\
\hline $\mathrm{P}(1)$ & $29(2)$ & $30(2)$ & $25(2)$ & $1(2)$ & 11(1) & $-2(2)$ \\
\hline $\mathrm{P}(2)$ & $29(2)$ & $28(2)$ & $30(2)$ & $1(2)$ & $14(1)$ & $-4(2)$ \\
\hline $\mathrm{P}(3)$ & $43(2)$ & $40(2)$ & $40(2)$ & $-2(2)$ & $11(2)$ & $-3(2)$ \\
\hline $\mathrm{F}(1)$ & $57(5)$ & $72(7)$ & $88(6)$ & $-8(5)$ & $5(4)$ & $-6(5)$ \\
\hline $\mathrm{F}(2)$ & $100(6)$ & $149(10)$ & $43(5)$ & $-22(6)$ & $36(5)$ & $-33(7)$ \\
\hline $\mathrm{F}(3)$ & $66(5)$ & $48(6)$ & 115(7) & $-6(5)$ & $42(5)$ & $6(5)$ \\
\hline $\mathrm{F}(4)$ & $66(5)$ & 119(8) & $58(5)$ & $3(6)$ & $44(4)$ & $21(5)$ \\
\hline $\mathrm{F}(5)$ & $88(6)$ & $48(6)$ & $110(7)$ & $21(5)$ & $16(5)$ & $7(5)$ \\
\hline $\mathrm{F}(6)$ & $43(4)$ & $57(5)$ & $53(5)$ & $-13(4)$ & $6(4)$ & $0(4)$ \\
\hline $\mathrm{N}(1)$ & $22(5)$ & $20(6)$ & $30(6)$ & $-7(5)$ & $11(5)$ & $2(5)$ \\
\hline $\mathrm{N}(2)$ & $23(5)$ & $37(6)$ & $14(5)$ & $2(5)$ & $5(4)$ & $-1(5)$ \\
\hline $\mathrm{O}(1)$ & $55(6)$ & $20(5)$ & $61(6)$ & $1(5)$ & $10(5)$ & $-14(5)$ \\
\hline $\mathrm{O}(2)$ & $50(5)$ & $37(6)$ & $33(5)$ & $14(5)$ & $15(4)$ & $4(5)$ \\
\hline $\mathrm{C}(1)$ & $35(7)$ & $37(9)$ & $24(7)$ & $10(7)$ & $-1(6)$ & $9(7)$ \\
\hline$C(2)$ & $21(6)$ & $19(7)$ & $45(8)$ & $-3(6)$ & $23(6)$ & $-2(5)$ \\
\hline$C(3)$ & $32(7)$ & $44(9)$ & $28(7)$ & $3(7)$ & $16(6)$ & $-2(6)$ \\
\hline$C(4)$ & $29(7)$ & $31(8)$ & $23(7)$ & $3(6)$ & $5(5)$ & $-4(6)$ \\
\hline$C(5)$ & $43(7)$ & $29(8)$ & $26(7)$ & $-7(6)$ & $8(6)$ & $-6(6)$ \\
\hline$C(6)$ & $35(7)$ & $62(10)$ & $35(7)$ & $2(7)$ & $26(6)$ & $-1(7)$ \\
\hline$C(7)$ & $30(8)$ & $53(10)$ & $65(10)$ & $-20(9)$ & $15(7)$ & $11(8)$ \\
\hline $\mathrm{C}(8)$ & $51(10)$ & $46(10)$ & $53(10)$ & $12(8)$ & $-8(8)$ & $6(8)$ \\
\hline $\mathrm{C}(9)$ & $38(9)$ & $53(11)$ & $70(10)$ & $10(9)$ & $11(8)$ & $-3(8)$ \\
\hline$C(10)$ & $44(8)$ & $24(8)$ & $55(9)$ & $14(7)$ & $22(7)$ & $7(7)$ \\
\hline $\mathrm{C}(11)$ & $16(6)$ & $20(7)$ & $45(8)$ & $9(6)$ & $5(6)$ & $-1(5)$ \\
\hline$C(12)$ & $21(6)$ & $48(10)$ & $30(7)$ & $-3(6)$ & $9(5)$ & $1(6)$ \\
\hline$C(13)$ & $54(9)$ & $54(11)$ & $37(8)$ & $4(7)$ & $27(7)$ & $4(8)$ \\
\hline$C(14)$ & $56(9)$ & $68(11)$ & $31(8)$ & $5(8)$ & $23(7)$ & $-7(8)$ \\
\hline$C(15)$ & $46(8)$ & $58(10)$ & $27(7)$ & $6(7)$ & $10(6)$ & $-5(7)$ \\
\hline$C(16)$ & $39(7)$ & $51(9)$ & $16(7)$ & $-1(6)$ & $-2(6)$ & $10(7)$ \\
\hline$C(17)$ & $21(6)$ & $36(8)$ & $24(7)$ & $-7(6)$ & $3(5)$ & $-13(6)$ \\
\hline$C(18)$ & $49(8)$ & $42(9)$ & $49(9)$ & $7(8)$ & $28(7)$ & $0(7)$ \\
\hline C(19) & $70(10)$ & $52(11)$ & $49(9)$ & $-1(8)$ & $31(8)$ & $-19(9)$ \\
\hline$C(20)$ & $61(10)$ & $47(10)$ & $37(9)$ & $2(8)$ & $3(8)$ & $-8(8)$ \\
\hline$C(21)$ & $41(8)$ & $35(9)$ & $50(9)$ & $0(8)$ & $13(7)$ & $6(7)$ \\
\hline
\end{tabular}




\begin{tabular}{rrrrrrr}
$\mathrm{C}(22)$ & $53(9)$ & $32(8)$ & $47(8)$ & $0(8)$ & $24(7)$ & $-7(7)$ \\
$\mathrm{C}(23)$ & $23(6)$ & $19(6)$ & $24(6)$ & $4(6)$ & $2(5)$ & $4(6)$ \\
$\mathrm{C}(24)$ & $38(7)$ & $26(7)$ & $21(7)$ & $4(6)$ & $1(6)$ & $-7(6)$ \\
$\mathrm{C}(25)$ & $24(6)$ & $25(7)$ & $33(7)$ & $-3(6)$ & $3(6)$ & $-8(6)$ \\
$\mathrm{C}(26)$ & $51(9)$ & $28(8)$ & $40(8)$ & $1(7)$ & $7(7)$ & $11(7)$ \\
$\mathrm{C}(27)$ & $62(8)$ & $23(7)$ & $32(7)$ & $1(7)$ & $18(6)$ & $-9(7)$ \\
$\mathrm{C}(28)$ & $35(7)$ & $29(7)$ & $24(7)$ & $-4(6)$ & $1(6)$ & $0(6)$ \\
$\mathrm{C}(29)$ & $31(7)$ & $41(8)$ & $24(7)$ & $4(6)$ & $11(6)$ & $-2(6)$ \\
$\mathrm{C}(30)$ & $47(8)$ & $40(9)$ & $37(8)$ & $1(7)$ & $23(7)$ & $5(7)$ \\
$\mathrm{C}(31)$ & $26(6)$ & $32(8)$ & $38(7)$ & $-10(6)$ & $16(6)$ & $1(6)$ \\
$\mathrm{C}(32)$ & $35(7)$ & $29(8)$ & $28(7)$ & $-1(6)$ & $11(6)$ & $3(6)$ \\
$\mathrm{C}(33)$ & $21(6)$ & $27(8)$ & $28(7)$ & $-12(6)$ & $5(6)$ & $0(6)$ \\
$\mathrm{C}(34)$ & $11(6)$ & $32(7)$ & $24(7)$ & $-1(6)$ & $4(5)$ & $-4(6)$ \\
$\mathrm{C}(35)$ & $26(6)$ & $29(8)$ & $25(6)$ & $-6(6)$ & $3(5)$ & $-1(6)$ \\
$\mathrm{C}(36)$ & $35(7)$ & $37(9)$ & $26(7)$ & $17(6)$ & $-6(6)$ & $-4(7)$ \\
$\mathrm{C}(37)$ & $40(8)$ & $34(8)$ & $30(7)$ & $6(7)$ & $5(6)$ & $11(7)$ \\
$\mathrm{C}(38)$ & $35(7)$ & $26(7)$ & $34(8)$ & $-2(6)$ & $12(6)$ & $1(6)$ \\
$\mathrm{C}(39)$ & $30(7)$ & $51(9)$ & $25(7)$ & $4(7)$ & $3(6)$ & $13(6)$ \\
$\mathrm{C}(40)$ & $36(7)$ & $35(8)$ & $31(7)$ & $8(6)$ & $4(6)$ & $21(6)$ \\
\hline $\mathrm{T}$ & & & &
\end{tabular}

The anisotropic displacement factor exponent takes the form: $-2 \pi^{2}\left[\mathrm{~h}^{2} \mathrm{a}^{2} \mathrm{U}_{11}+\ldots+\right.$ $2 \mathrm{hka} * \mathrm{~b} * \mathrm{U}_{12}$ ] 
Table S12. Hydrogen coordinates $\left(\times 10^{4}\right)$ and isotropic displacement parameters $\left(\AA^{2} \times 10^{3}\right)$ for $\left[\operatorname{Re}(\right.$ phen $)($ dppe $\left.)(\mathrm{CO})_{2}\right]\left(\mathrm{PF}_{6}\right)$.

\begin{tabular}{|c|c|c|c|c|}
\hline Atom & $\mathrm{x}$ & $\mathrm{y}$ & $\mathrm{z}$ & $\mathrm{U}(\mathrm{eq})$ \\
\hline $\mathrm{H}(3)$ & 8803 & 1921 & 9316 & 40 \\
\hline $\mathrm{H}(4)$ & 8607 & 3127 & 9979 & 34 \\
\hline $\mathrm{H}(6)$ & 8898 & 469 & 8812 & 48 \\
\hline $\mathrm{H}(7)$ & 9727 & -813 & 9221 & 60 \\
\hline $\mathrm{H}(8)$ & 9253 & -1761 & 10055 & 68 \\
\hline $\mathrm{H}(9)$ & 7904 & -1541 & 10389 & 67 \\
\hline $\mathrm{H}(10)$ & 7043 & -293 & 9957 & 48 \\
\hline $\mathrm{H}(12)$ & 7796 & 2312 & 7785 & 40 \\
\hline $\mathrm{H}(13)$ & 7274 & 2335 & 6298 & 54 \\
\hline $\mathrm{H}(14)$ & 6243 & 1312 & 5533 & 59 \\
\hline $\mathrm{H}(15)$ & 5799 & 206 & 6213 & 53 \\
\hline $\mathrm{H}(16)$ & 6323 & 104 & 7687 & 45 \\
\hline $\mathrm{H}(18)$ & 7315 & 3756 & 8490 & 52 \\
\hline H(19) & 6643 & 4932 & 7617 & 65 \\
\hline $\mathrm{H}(20)$ & 5512 & 5744 & 7873 & 62 \\
\hline $\mathrm{H}(21)$ & 5044 & 5434 & 9035 & 51 \\
\hline $\mathrm{H}(22)$ & 5716 & 4271 & 9923 & 51 \\
\hline $\mathrm{H}(24)$ & 8263 & 4226 & 10897 & 37 \\
\hline $\mathrm{H}(25)$ & 8799 & 4674 & 12314 & 35 \\
\hline $\mathrm{H}(26)$ & 8129 & 4086 & 13243 & 50 \\
\hline $\mathrm{H}(27)$ & 6988 & 3035 & 12830 & 46 \\
\hline $\mathrm{H}(28)$ & 6485 & 2565 & 11408 & 38 \\
\hline $\mathrm{H}(29)$ & 5111 & 1669 & 10941 & 38 \\
\hline $\mathrm{H}(30)$ & 3914 & 2361 & 11288 & 47 \\
\hline $\mathrm{H}(31)$ & 3017 & 3398 & 10371 & 37 \\
\hline $\mathrm{H}(36)$ & 3702 & 3868 & 6651 & 44 \\
\hline $\mathrm{H}(37)$ & 4781 & 2956 & 6406 & 44 \\
\hline $\mathrm{H}(38)$ & 5666 & 2052 & 7472 & 38 \\
\hline H(39) & 2601 & 4149 & 8940 & 44 \\
\hline $\mathrm{H}(40)$ & 2896 & 4387 & 7709 & 43 \\
\hline
\end{tabular}




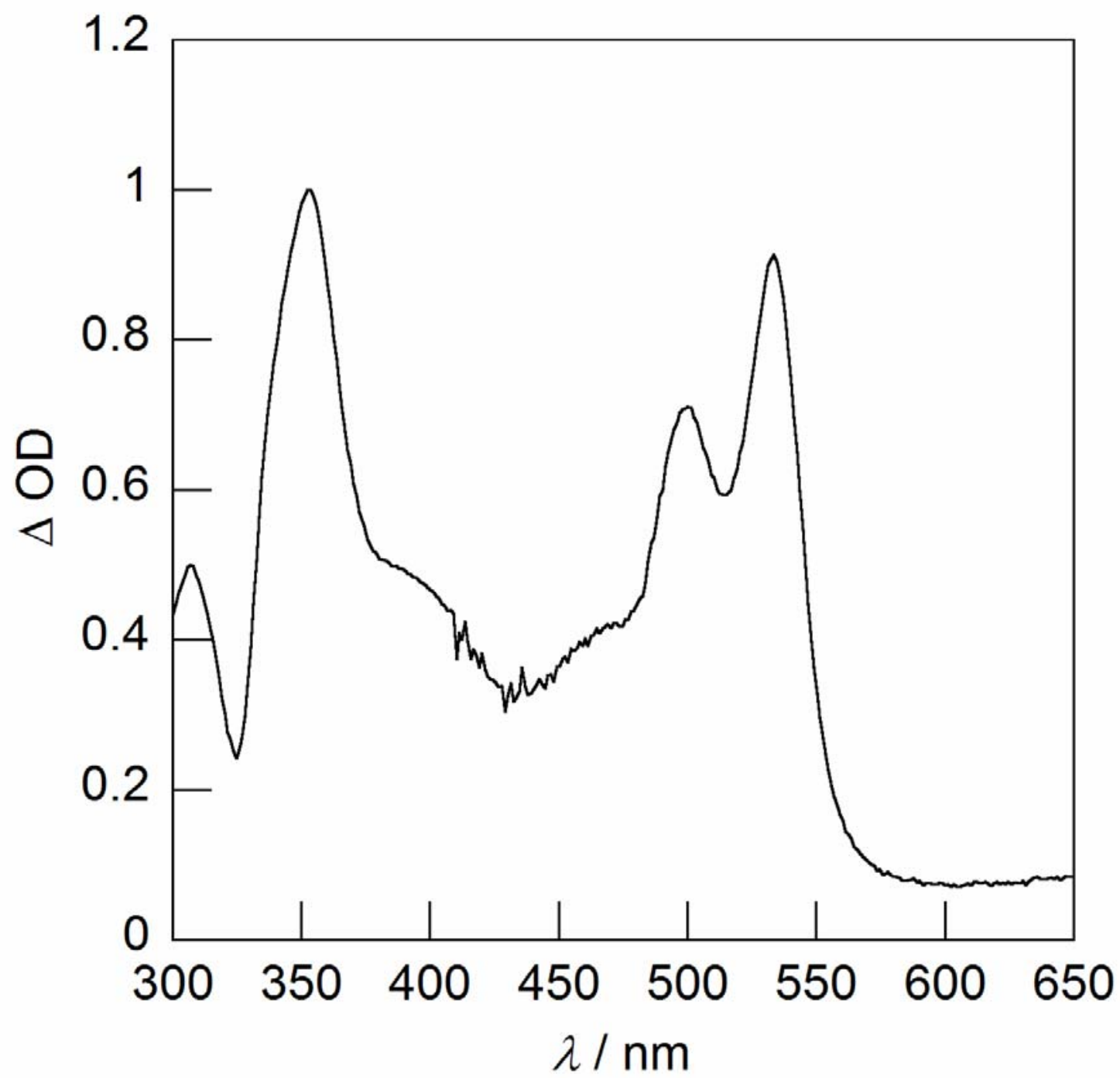

Figure S3. Change in absorbance following electrolysis of $\mathrm{Re}(\mathrm{bpy}-\mathrm{Y}-\mathrm{O} t \mathrm{Bu})(\mathrm{CO})_{3}(\mathrm{CN})$ at -0.75 $\mathrm{V}$ vs. $\mathrm{Fc}^{+} / \mathrm{Fc}$ in $\mathrm{DMF}$ with $0.1 \mathrm{M} \mathrm{TBA}\left(\mathrm{PF}_{6}\right)$ supporting electrolyte corresponding to the reduced $\mathrm{Re}\left(\mathrm{bpy} \bullet^{-}-\mathrm{Y}-\mathrm{O} t \mathrm{Bu}\right)(\mathrm{CO})_{3}(\mathrm{CN})$. 